\title{
The design of a multilevel envelope tracking amplifier based on a multiphase buck converter
}

\author{
M. Vasić, P. Cheng, O. García, J. A. Oliver, P. Alou, J. A. Cobos, \\ Universidad Politécnica de Madrid, Centro de Electrónica Industrial \\ D. Tena, F. J. Ortega \\ Universidad Politécnica de Madrid, Radio Engineering Group (GIRA) \\ Corresponding author: Miroslav Vasić \\ E-mail: miroslav.vasic aupm.es
}

\begin{abstract}
Envelope Tracking (ET) and Envelope Elimination and Restoration (EER) are techniques that have gained in importance in the last decade in order to obtain highly efficient Radio Frequency Power Amplifier (RFPA) that transmits signals with high Peak to Average Power Ratio (PAPR). In this work a multilevel multiphase buck converter is presented as a solution for the envelope amplifier used in ET and EER. The presented multiphase buck converter generates multilevel voltage using "node" duty cycles and non-linear control. In this way the multilevel is implemented using only one simple power stage. However, the complexity of the multilevel converter implementation has been shifted from complicated power topologies to complicated digital control. Detailed discussion regarding the influence of the design parameters (switching frequency, output filter, time resolution of the digital control) on the performance of the proposed envelope amplifier is presented. The design of the output filter is conducted fulfilling the constraints of the envelope slew rate and minimum driver pulse that can be reproduced. In the cases when these two constraints cannot be fulfilled, they may be relieved by the modified control that is presented and experimentally validated. Finally, in order to validate the concept, a prototype has been designed and integrated with a nonlinear class $\mathrm{F}$ amplifier. Efficiency measurements showed that by employing EER it is possible to save up to $15 \%$ of power losses, comparing to the case when it is supplied by a constant voltage. Additionally, Adjacent Channel Power Ratio (ACPR) has been measured. The obtained results showed the value higher than $30 \mathrm{~dB}$ for signals up to $5 \mathrm{MHz}$ of bandwidth, without using predistortion technique.
\end{abstract}

Keywords: Envelope Tracking, Envelope Elimination and Restoration, Multilevel converter, Multiphase converter

\section{INTRODUCTION}

The requirement of delivering high data rates in modern wireless communication systems results in complex modulated RF signals with wide bandwidth and high PAPR. In order to guarantee high linearity of the power amplifier (PA), the conventional linear power amplifiers typically operate at $4 \mathrm{~dB}$ to $10 \mathrm{~dB}$ back-off from the maximum output power, leading to low system efficiency. There are several techniques to overcome this efficiency problem such as 
Doherty amplifier [1], Outphasing amplifier [2], Envelope Tracking amplifier (ET) and Envelope Elimination and Restoration amplifier (EER) [3, 4]. ET and EER are two promising techniques for improving the efficiency and the linearity of PAs that have recently received significant attention. In both ET and EER, the transmitter is composed of an RF PA and an Envelope Amplifier (EA) that supplies the RF PA following the envelope of the transmitted signal as shown in Figure 1. The design of the envelope amplifier is crucial for the system performance. State of the art presents many research activities on this topic, dividing the research lines according:

- fast tracking

- reduced bandwidth tracking

- multilevel tracking
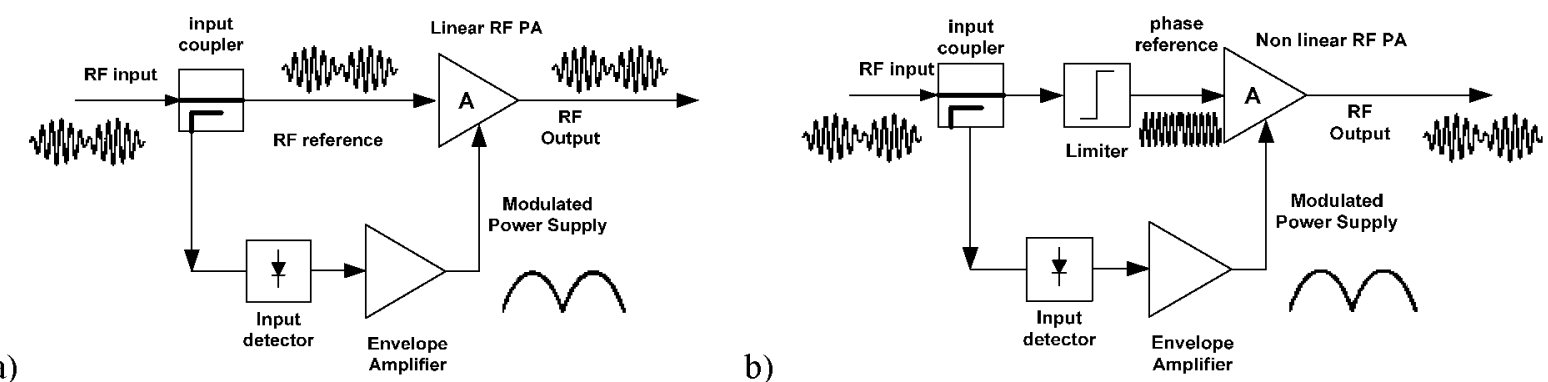

Fig. 1. Simplified block schematics of radio transmitters based on: a) ET and b) EER technique

Fast tracking methods are identified as the solutions where output of the EA exactly follows the RF signal envelope. In this way, in an ideal case, the efficiency of the RF PA can be maximized, since it is always working at the point where the envelope amplitude voltage is equal to supply voltage. However, the system efficiency depends not only on the RF PA efficiency, but also on the efficiency of the envelope amplifier itself. The challenge for the EA design is to obtain the wide bandwidth together with high PAPR of the transmitted RF signal. A fast tracking EA usually is implemented as a high switching frequency converter or a switch-mode assisted linear amplifier. A switching converter used as an EA, usually is a buck type converter which needs to operate at very high switching frequency in order to accurately track the envelope. In this way, it is guaranteed that signal's bandwidth is wider than the envelope's bandwidth, which is crucial for achieving high linearity of the transmitter.

The EA switching frequency does not penalize the overall efficiency in the case of a low bandwidth envelope tracking such as in [6], where a buck converter switches at $200 \mathrm{kHz}$ to track a $10 \mathrm{kHz}$ envelope. However in [7] and [8], when EA has to track $20 \mathrm{MHz}$ and $15 \mathrm{MHz}$ envelopes, the reported switching frequencies are $200 \mathrm{MHz}$ and $130 \mathrm{MHz}$ respectively. For such a high frequency, it is necessary to implement an EA with integrated circuits, because the parasitic PCB inductances have serious impacts on the converter's performance and the overall efficiency [9]. On the other hand, the integrated circuits can significantly reduce these parasitic elements, but limiting the level of the output power due to the thermal issues. To overcome this problem, EAs may be implemented as a switch-mode assisted linear amplifier that 
is introduced in [10-13]. A typical structure includes a switching stage and a linear stage connected in parallel. The low frequency parts of the load current pass through the switching stage, so that the high switching frequency is not needed, while the high frequency parts pass through the linear stage. The bandwidth separation point can be adjusted and an optimal band separation can be obtained using the envelope amplitude density distribution $[14,15]$. However, with the envelope bandwidth and PAPR increasing, this type of the EA may have problems with low efficiency since the switching stage is not able to handle such a fast current change, leading to a big portion of the current flow through the linear stage. In [16] two switching stages the main switching stage (high frequency) and auxiliary switching stage (low frequency) are introduced in order to improve the efficiency under this condition. In the case when the highly efficient switching converter cannot follow the desired slew rate, an additional current source in parallel can be used like in [17]. However, this requires a lot of hardware, which is not practical unless it is integrated like in [17].

The output of an EA can also track the "slow version" of the RF signal envelope $[18,19]$ in order to reduce the energy consumed by a power amplifier system. This technique is called "reduced bandwidth tracking" and, although, it is originally developed for ET, it can also be applied to EER by adding a fast linear amplifier before the PA to shape the supply waveform into the envelope. By tracking a slow envelope, the switching converter in EA can operate in more relaxed conditions, thus its efficiency can be increased. However, it is at the expense of reducing the efficiency of the $\mathrm{PA}$, since the PA is not in compression region all the time. In [20], the method of slow envelope generation is related to the filtering the original envelope using a low pass filter and then compensating the "chopped" part of the waveform. Consequently, the overall system efficiency remains high. In [21] and [22] the slow envelopes have limited slew rate due to the restriction of the switching converter. The key point of reduced bandwidth tracking is to have power loss trade-off between EA and PA, which makes even more sense for amplifying RF signals whose envelope bandwidth is beyond the reach of available efficient EA circuits.

The multilevel voltage tracking is a concept similar to the reduced bandwidth tracking. Instead of using a smooth waveform, it provides discrete voltage levels which are intended to be as close as possible to the output voltage of the RF PA. The multilevel voltage tracking behaves like a power analog to digital converter, but it has to guarantee that the supply voltage is instantaneously higher than the amplified signal envelope. This approach is proposed in [23] and enhanced by $[24,25]$. In [24], multilevel voltages are generated by stacked voltage cells which are supplied by a multiple-output flyback DC-DC converter stage. In [25] switching capacitor based voltage divider is used as a multilevel voltage circuit, which shows better efficiency than the stacked cells solution. The cells of the multilevel converter can be used in order to implement PWM multilevel buck converter [26]. Due to the lower voltages that are applied on the output filter, its size can be decreased and the large signal bandwidth can be improved. However, the bandwidth of the converter 
remains a fraction of the switching frequency, like in any other linear control approach. Nevertheless, in [27, 28] it has been shown that in the case of perfect filtering of the PWM signal, it might be possible to use switching frequency quite close to the desired bandwidth, at least from the point of view of theoretical discussion. Certain improvements from the point of view of the ratio between the converter's large signal bandwidth and its switching frequency are obtained by complex design of the output filter presented in $[29,30]$.

In [31], a concept of multiphase buck converter with minimum time control for multilevel voltage tracking is presented and validated with a 4-phase converter. The basic idea of the multilevel converter in [31] is shown in Figure 2. The EA is composed of a multiphase buck converter which operates exclusively at "node" duty cycles. This means that in an ideal case the current ripple does not exist and that it is possible to decrease the values of the inductance and capacitance in the output filter. The converter where the output filter has low inductance and capacitance may be able to follow fast dynamic signals, due to lower "inertia" of the system. In a hypothetical case, the output capacitance could be totally eliminated. Each phase of the converter operates with high current ripple (critical conduction mode) and in that way the current self-balance is obtained and current sharing can be guaranteed [32]. The converter's "node" operation brings another important characteristic. The number of voltage levels that it can produce is equal to the number of phases. The proposed multilevel converter generates the needed voltage levels by selecting the duty cycle between the limited set of values. In order to change the output voltage as fast as possible between two voltage levels, nonlinear minimum time transient control is used. During the transient, each transistor in each phase is turned on (with a duration $t_{\text {on }}$ ) and turned off (with a duration $t_{\text {off }}$ ) only once in order to reach the desired output voltage in the shortest possible time Figure 3 illustrates the minimum transition time in a case of a 4 phase buck converter. By implementing this concept the implementation of the multilevel converter can be done using only one simple power stage and this is not the case in the solutions presented in [23-25]. It does not need any complicated hardware like in [17] and it will be shown that its performance is not sensitive to component tolerances which is not the case in the solutions presented in [29, 30] However, since the concept of the generation of the different voltage levels has moved from power architecture to the control, the required non-linear control is significantly more complex. This concept is presented in detail in [31] and this paper presents the design and optimization of the multiphase multilevel converter for wide bandwidth signals.

The reproduced envelopes are for the signals that are for an order of magnitude faster than in [31] and due to high dynamics the design of the converter face limitations due to transistor's driver nonidealities and constraints. In order to cope with this issue, two novel control strategies have been introduced and verified with experimental results: phase reshuffle after voltage transitions and transition shifts. With these techniques the designed multiphase multilevel converter was possible to follow signals up to $10 \mathrm{MHz}$ of bandwidth. Finally the implemented multilevel converter was 
integrated with a nonlinear RF PA and series of measurements (linearity and efficiency) were performed in order to validate the concept.

In the case of ET only the proposed multilevel converter can supply the RF PA, while in the case of the EER it is necessary to combine the multilevel in series with a linear regulator so that the exact replica of the envelope is reproduced and supplied to the RF PA. In this paper, it is considered that the multilevel is always combined with the linear regulator (envelope amplifier for an EER transmitter), unless it is otherwise stated.

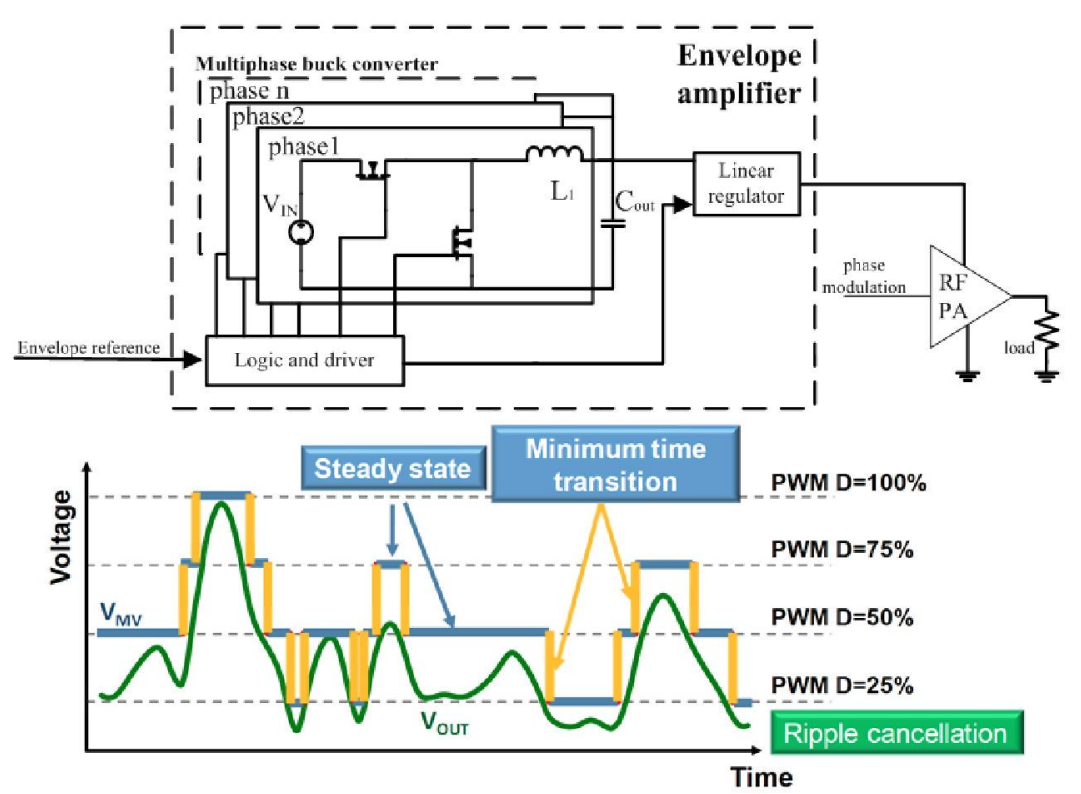

Fig. 2. Simplified schematic of the envelope amplifier based on a multiphase multilevel converter in series with a linear regulator

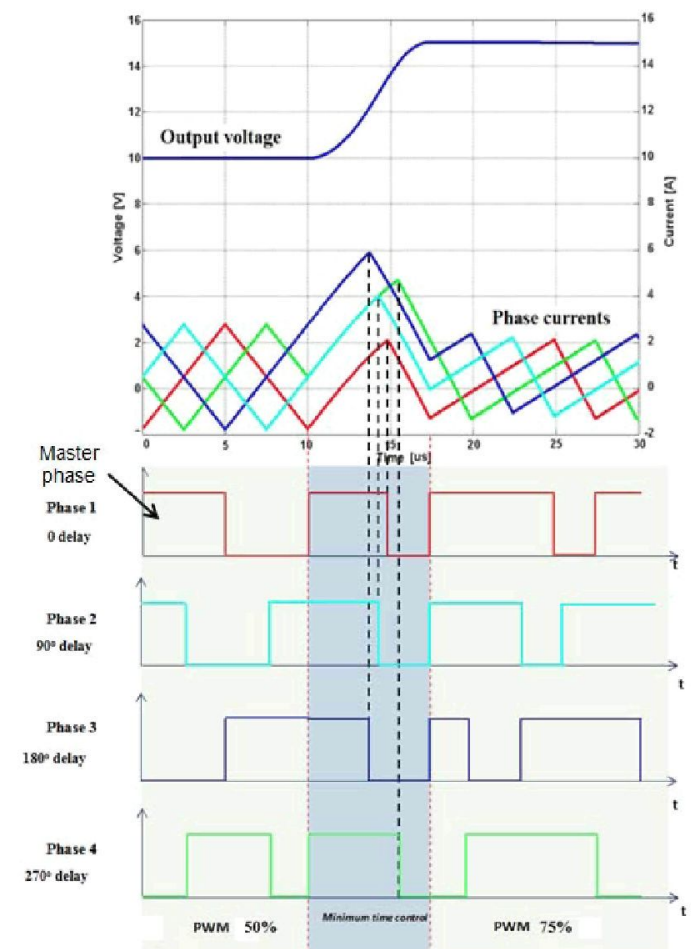

Fig. 3. Minimum time transient in a case of a 4 phase buck converter. Output voltage, phase currents and gate signals for each phase are presented 
The previously discussed topology, multiphase buck converter that operates in "node" duty cycles and performs minimum transition time, will be analyzed in detail in this section. The design of the output filter will be explained, as well as, how to select the optimal switching frequency. The presented analysis has been conducted for an 8 phase buck converter and maximal output voltage of $42 \mathrm{~V}$. The signal with the bandwidth of $5 \mathrm{MHz}$ will be used as the reference signals in the analysis of the envelope tracker performance.

\section{A. Output filter design area}

For wide bandwidth envelope applications, short transition time of the proposed multilevel converter is required due to the fast tracking. This transition time is implemented using Minimum Time Control (MTC) and it mainly depends on the filter's value (L and C). Theoretically, smaller filter means less energy storage for necessary change of the state and it naturally means less transition time. According to the model in [31], the transition time can be as small as possible if the filter is small enough. In [31] it was suggested that the output filter should be designed so that the multilevel converter's slew rate is faster than the envelope's slew rate. Nevertheless, when wide bandwidth signals are transmitted, the ability of real hardware to correctly generate the minimum voltage pulse is limited. For the given number of phases of the multiphase converter, in order to design the output filter, two constraints have been taken into account:

- The minimum slew rate constraint (specified by the application, i.e. the signal that is transmitted)

- The minimum voltage pulse duration constraint (specified by the transistor driver and minimum clock period of the digital control)

During the steady state (constant duty cycle), by setting a proper threshold voltage for the multilevel converter, the converter's output can always stay higher than the envelope amplitude, which is important in order to avoid the clipping of the RF PA output voltage. During the transition, in order to avoid the supply voltage cutting the envelope as shown in Figure 4, the slew rate of converter's output voltage $\left(\mathrm{SR}_{\mathrm{MTC}}\right.$, Slew Rate Minimum Time Control) is designed to be higher than the slew rate of the envelope $\left(\mathrm{SR}_{\mathrm{E}}\right)$. $\mathrm{SR}_{\mathrm{MTC}}$ can be expressed as:

$$
S R_{M T C}=\frac{V_{2}-V_{1}}{\Delta t}
$$

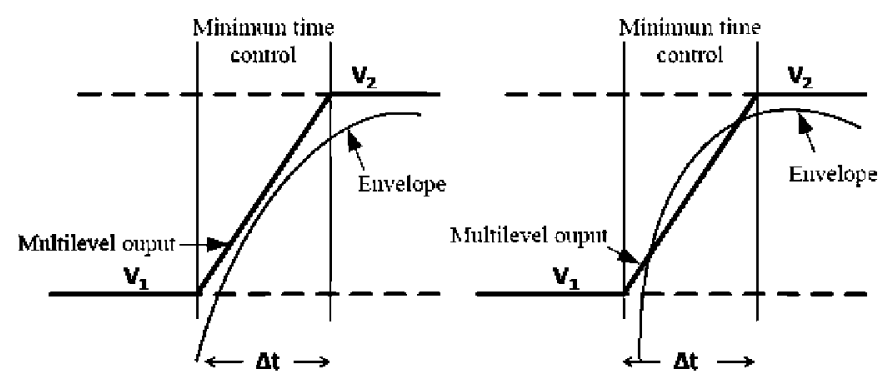

Fig. 4. A case when the slew rate of the envelope is lower than the slew rate of converter's output (left) and the opposite case (right) during the transition. 
where $V_{1}$ is the output voltage before the transient, $V_{2}$ the output voltage after the transient and $\Delta t$ the duration of the transient. $\mathrm{SR}_{\mathrm{E}}$ generally increases with the increase of the envelope bandwidth. The worst case happens when the minimum $\mathrm{SR}_{\mathrm{MTC}}$ is equal to the maximum $\mathrm{SR}_{\mathrm{E}}$. For a particular application where the input voltage and the number of phase are fixed, $\mathrm{SR}_{\mathrm{MTC}}$ depends on the transition voltage $\left(\mathrm{V}_{2}-\mathrm{V}_{1}\right)$ and transition time $(\Delta \mathrm{t})$. The analysis presented in [31] shows how to derive the following expressions for $\Delta \mathrm{t}$ (transition time), $\mathrm{t}_{\mathrm{ON}, \mathrm{i}}\left(\mathrm{ON}\right.$ time in the $\mathrm{i}^{\text {th }}$ phase) and $\mathrm{t}_{\mathrm{OFF}, \mathrm{I}}(\mathrm{OFF}$ time in the $i^{\text {th }}$ phase):

$$
\begin{gathered}
t_{\text {on }, i}=\frac{V_{1}+V_{2}}{2 V_{\text {in }}} \Delta t+\frac{L \Delta I_{i}}{V_{\text {in }}} \\
\Delta t^{2}\left(-\frac{V_{\text {in }}}{2 L} N K^{2}+\frac{V_{\text {in }}}{L} N K-\frac{V_{1}}{2 L} N-\frac{V_{2}-V_{1}}{6 L} N\right)=C \Delta V+\frac{L}{2 V_{\text {in }}} \sum_{\mathrm{i}=1}^{\mathrm{N}} \Delta I_{i}^{2} \quad K=\frac{V_{1}+V_{2}}{2 V_{\text {in }}} \\
t_{\text {off }, i}=\Delta t-t_{\text {on }, i}
\end{gathered}
$$

where $\mathrm{L}$ and $\mathrm{C}$ are the values of the $\mathrm{LC}$ filter, $\mathrm{N}$ number of phases, $\Delta \mathrm{I}_{\mathrm{i}}$ is the difference of the phase current after and before the transient. It is important to notice that $\Delta \mathrm{t}$ is the same for each phase, while $\mathrm{t}_{\mathrm{ON}}$ and $\mathrm{t}_{\mathrm{OFF}}$ per phase differs. These times are related to the filter values, $\mathrm{V}_{1}, \mathrm{~V}_{2}$, phase shifting and also the switching frequency. This means that $\mathrm{t}_{\mathrm{ON}}$ and $\mathrm{t}_{\mathrm{OFF}}$ are different not only among phases, but also for different voltage level transitions. Therefore, by analyzing all the possible voltage transitions in an $\mathrm{N}$ phase buck converter, it can be concluded that the transition duration differs depends on the initial and final voltage values, $V_{1}$ and $V_{2}$, and not only on the voltage $V_{2}-V_{1}$.

In the presented solution the output filter is designed in a way that the switching converter follows the envelope slew rate, similar to the analysis in [33]. Nevertheless, in [33] the switching converter is used along with the classical linear control (the filter is designed having in mind the desired bandwidth and phase delay), while the control in the presented solution is strictly nonlinear, and the filter design approach is, therefore, different.

On the other hand, the envelope also exhibits different slew rate as a function of the envelope amplitude due to its generation mechanism. Therefore, it is necessary to analyze the maximum envelope slew rate during each transition of the voltage level, as shown in Figure 5. 


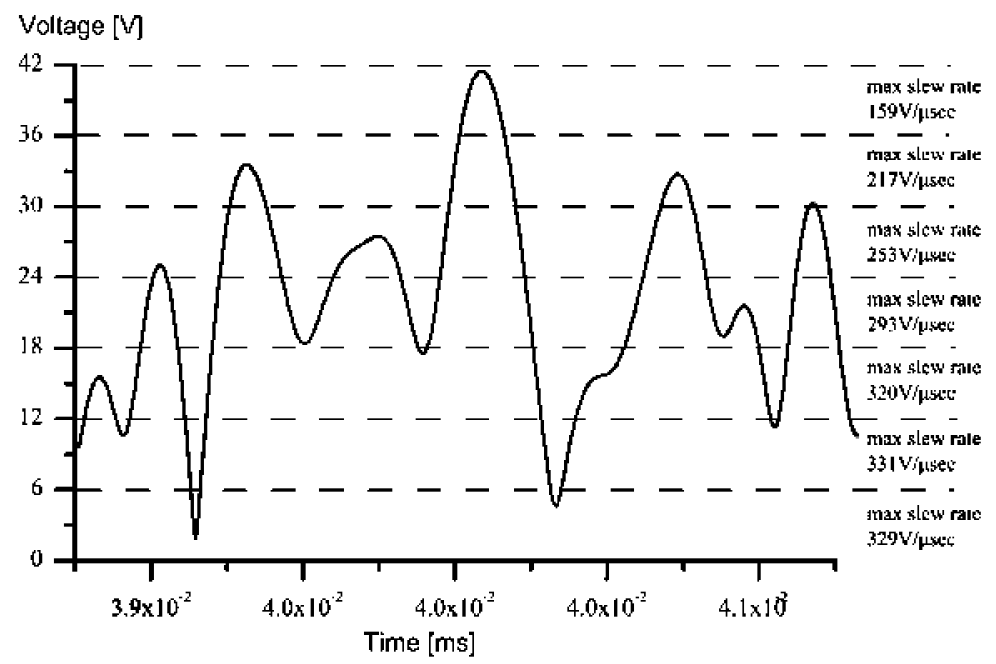

Fig. 5. The maximum slew rate at different voltage levels of a $10 \mathrm{MHz}$ bandwidth 64QAM envelope waveform. In this analysis it is assumed that the multilevel converter can produce 8 voltage levels

Previously presented analysis yields to an algorithm that is used to find filter design area based on the minimum slew rate constraint. It is a "brute force" algorithm that sweeps $\mathrm{L}$ and $\mathrm{C}$ values independently inside a certain design space. For a selected LC pair, a $\Delta \mathrm{t}$ for all possible voltage transitions is calculated, giving the information regarding the converter slew rate. If the calculated $\mathrm{SR}_{\mathrm{MTC}}$ is higher than the corresponding maximum $\mathrm{SR}_{\mathrm{E}}$, these $\mathrm{L}$ and $\mathrm{C}$ values are inside the design area and vice versa. Figure 6 illustrates the implemented algorithm for the design of the output filter in the case of the slew rate constraint.

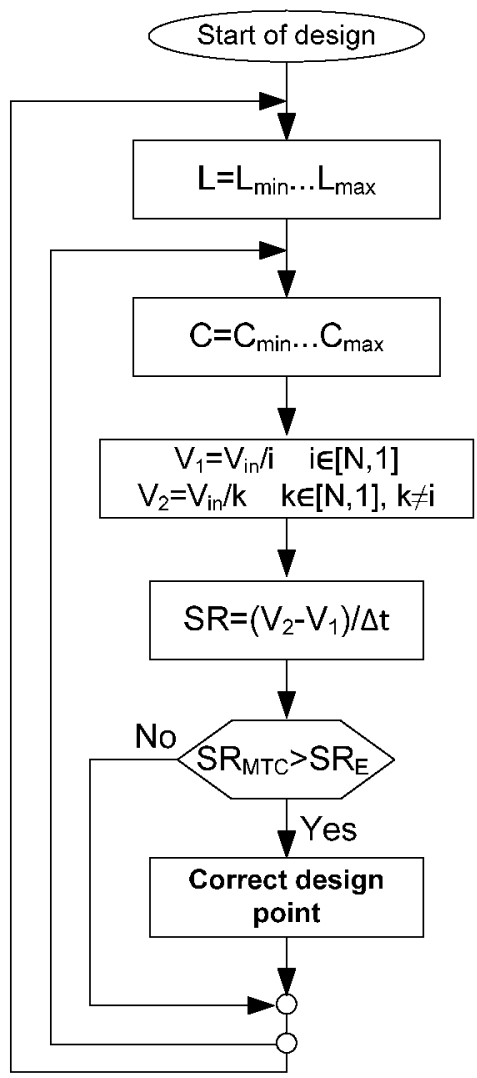

Fig. 6. Algorithm for the design of the output filter in the case of the output voltage slew rate constraint 
In Figure 7 a design area for the output filter in the case of the 8 phase buck converter for a $5 \mathrm{MHz}$ signal is shown. Smaller filter means smaller transition time, $\Delta t$, that is composed of a single $t_{\mathrm{ON}, \mathrm{i}}$ and $\mathbf{t}_{\mathrm{OFF}, \mathrm{i}}$ for each phase.

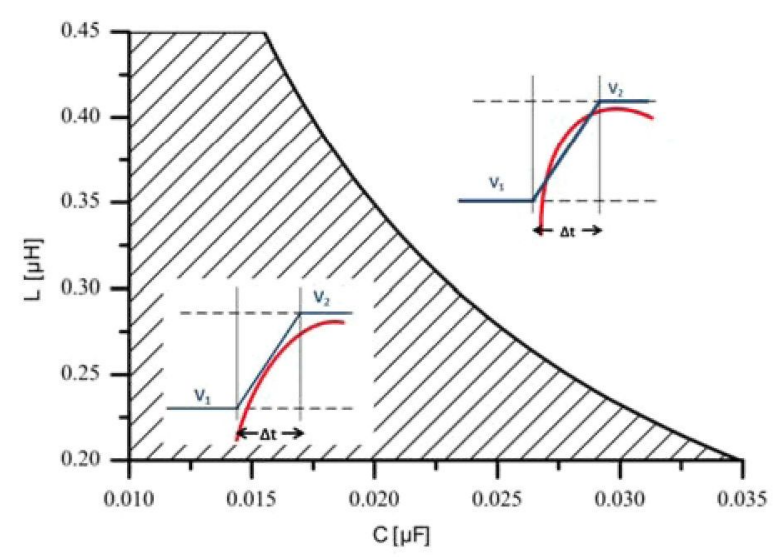

Fig. 7. LC design area for the minimum slew rate constraint in the case of the 8 phase converter and $5 \mathrm{MHz}$ signal

In the extreme cases, such as the step-up transitions which include the lowest voltage level $V_{\mathbb{I N}} / N_{\text {phase}}$, where $N_{\text {phase }}$ is the number of phases, short $\mathrm{t}_{\mathrm{ON}}$ and long $\mathrm{t}_{\mathrm{OFF}}$ usually are observed. On the other hand, the step-down transitions which include the highest voltage level $\left(\mathrm{V}_{\mathrm{IN}}\right)$ usually have long $\mathrm{t}_{\mathrm{ON}}$ and short $\mathrm{t}_{\mathrm{OFF}}$. With $\Delta \mathrm{t}$ becoming short, both $\mathrm{t}_{\mathrm{ON}, \mathrm{i}}$ and $\mathrm{t}_{\mathrm{OFF}, \mathrm{i}}$ can exceed the shortest width of pulse that the hardware (PWM modulator and transistor driver) can reproduce. Table I shows the minimum pulse duration needed in the transition obtained by the calculations for different application bandwidths.

TABLE I: The minimum pulse width of 8 voltage levels example, $V_{\text {out }}=42 \mathrm{~V}$

\begin{tabular}{|c|c|c|}
\hline Envelope bandwidth & The maximum slew rate & The minimum pulse width \\
\hline $2 \mathrm{MHz}$ & $\approx 78 \mathrm{~V} / \mu \mathrm{s}$ & $7 \mathrm{~ns}$ \\
\hline $5 \mathrm{MHz}$ & $\approx 162 \mathrm{~V} / \mu \mathrm{s}$ & $1.5 \mathrm{~ns}$ \\
\hline $10 \mathrm{MHz}$ & $\approx 331 \mathrm{~V} / \mu \mathrm{s}$ & $0.3 \mathrm{~ns}$ \\
\hline
\end{tabular}

Since the MOSFET driver normally sinks/sources limited current and because it needs to drive the impedance composed of gate resistance, gate-source and gate-drain capacitance, the gate-source pulse deviates from the ideal form and cannot be as short as we would like. There are always delays and limited slew rates of rising/falling edges. Therefore, it is not possible to respond to an extremely fast and short pulse, because there is a minimum time interval needed to switch on/off the MOSFET. Figure 8 shows an example of this situation with a fast driver (EL7158ISZ from Intersil) supplied by $5 \mathrm{~V}$ and MOSFET FDS89141 from Fairchild. When the width of the control is $10 \mathrm{~ns}$, the gate-source signal can reproduce the control signal, but with a delay. Due to the delay in the driver and the gate-source capacitor, when the width of the control pulse is $5 \mathrm{~ns}$, the gate-source signal is not able to respond. 


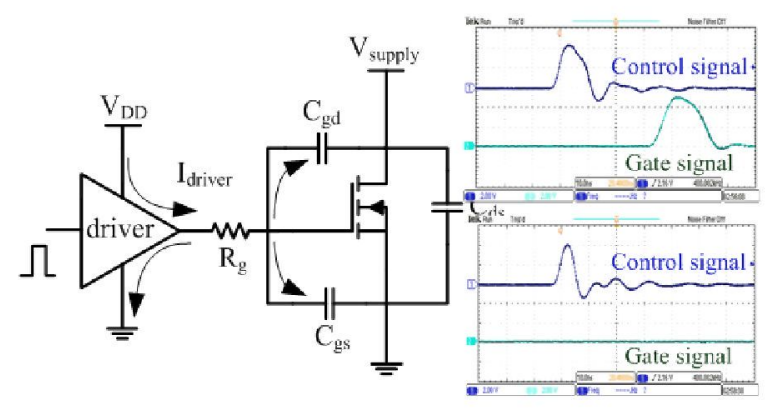

Fig. 8. An example of hardware limitation constraint.

Therefore, this hardware limitation sets the minimum value for $t_{\mathrm{ON}}$ and $\mathrm{t}_{\mathrm{OFF}}$ and has influence on the design of the multiphase output filter. Using this constraint, another LC design area is defined, which is the area above the borderline shown in Figure 9. Figure 9 shows the design space for $\mathrm{L}$ and $\mathrm{C}$ of the output filter if only the duration of the minimum voltage pulse is taken into account. The shown area corresponds to the same design case like in the case of Figure 7 (8 phase buck converter, $5 \mathrm{MHz}$ signal). Larger $\mathrm{L}$ or $\mathrm{C}$ values correspond to longer transient time, which relaxes the minimum pulse during $\Delta t$. An algorithm similar to the minimum slew rate constraints is used to identify the aforementioned borderline. By sweeping $\mathrm{L}$ and $\mathrm{C}$ value independently once again, the $\mathrm{t}_{\mathrm{ON}, \mathrm{i}}$ and $\mathrm{t}_{\mathrm{OFF}, \mathrm{i}}$ for each phase among all the possible transition levels are calculated. If all the interval times are longer than the minimum pulse width, the hardware is able to reproduce the control signals correctly and these $\mathrm{L}$ and $\mathrm{C}$ values are in the design area and vice versa. Figure 10 shows the filter design area for both the minimum slew rate constraint and the hardware limitation constraint (the minimum driver pulse width of $1 \mathrm{~ns}$ ). It can be observed that the overlap area is selected as the design area that can fulfil both constraints at the same time.

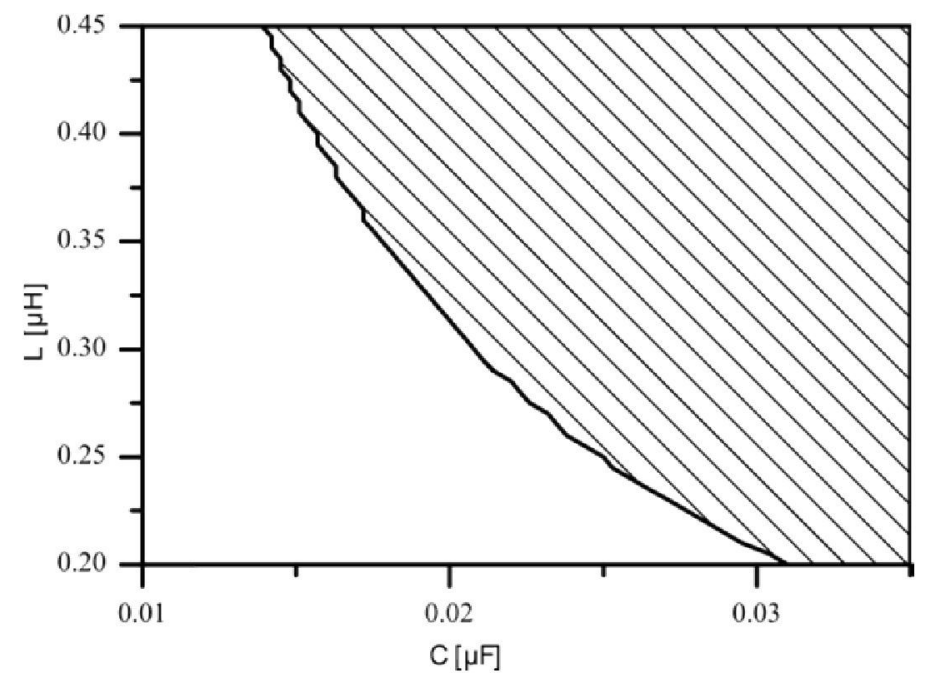

Fig. 9. LC design area for the minimum gate pulse duration costraint ( $1 \mathrm{~ns}$ in this case $)$ in the case of the 8 phase converter and a $5 \mathrm{MHz}$ signal 


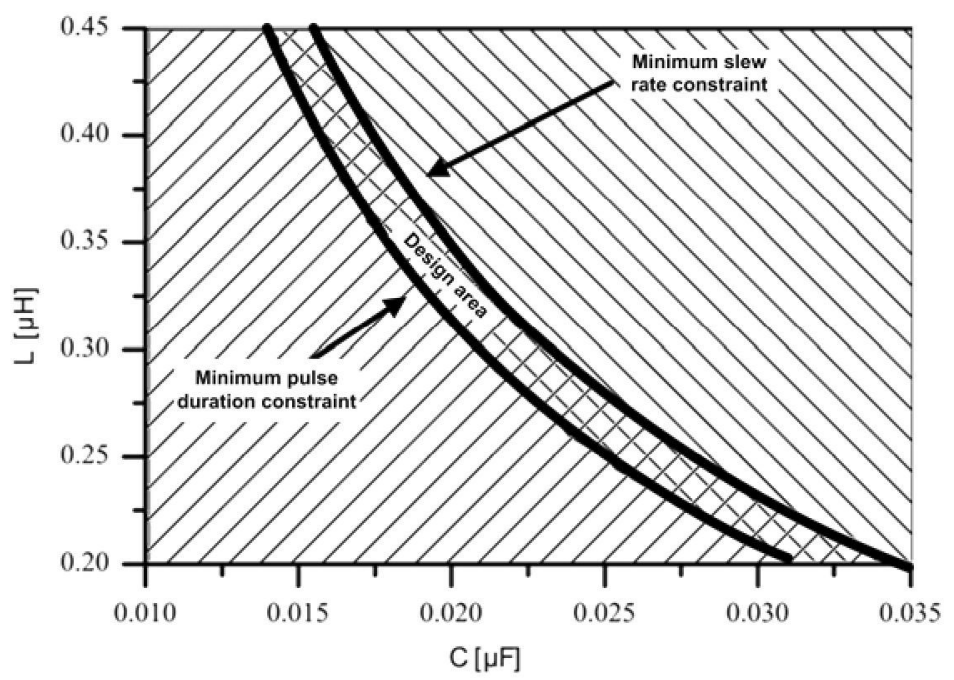

Fig. 10. LC design area for the minimum slew rate constraint and driver limitation constraint in the case of the 8 phase converter and a $5 \mathrm{MHz}$ signal

The design area in the case of a driver with minimum pulse width of $1 \mathrm{~ns}$ is obtained without the transitions that include the highest voltage level. The transition that includes the top level $\left(V_{\mathrm{IN}}\right)$ is different from the others. Only high side MOSFET is on at the top level and there is no current ripple. Considering step-up transition as an example, all the phase currents end up with DC value after the transient. $\Delta \mathrm{I}_{\mathrm{i}}$ has a higher value than in other cases. As it has been explained, large $\Delta \mathrm{I}_{\mathrm{i}}$ pushes $\mathrm{t}_{\mathrm{ON}, \mathrm{i}}$ to be more dominated in $\Delta \mathrm{t}$. On other hand, it forces the use of larger values of $\mathrm{L}$ and $\mathrm{C}$ due to the hardware limitation constraint, and moves the corresponding LC border to the right side. Figure 11 shows that the design space for the LC filter does not exist when the top level is used. Therefore, the solution is to remove the transitions that include the top level that penalizes the hardware limitation and the input voltage $\left(\mathrm{V}_{\mathrm{IN}}\right)$ is increased in order to cover the entire voltage range of the envelope. Due to this, the designed $\mathrm{N}$ phase converter will reproduce only $\mathrm{N}-1$ voltage levels and, hence, its supply voltage will be higher than the maximal output voltage.

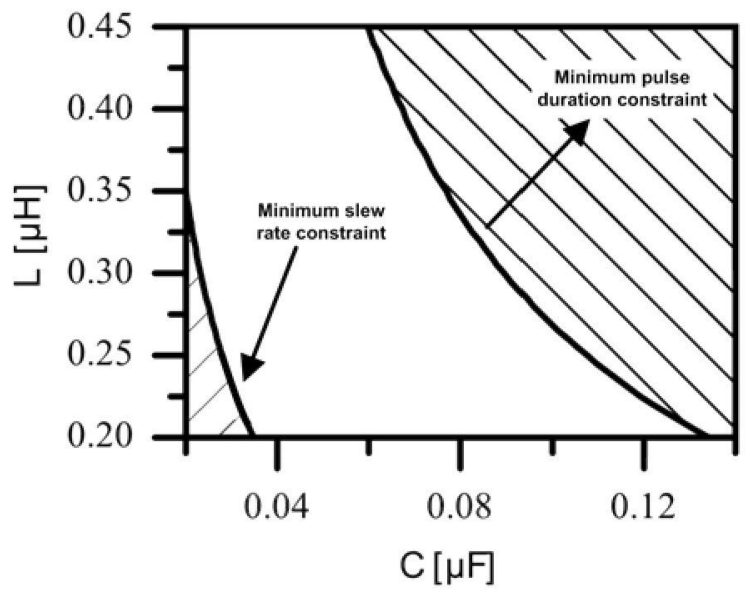

Fig. 11. LC design area including the highest voltage level in the case of a $5 \mathrm{MHz}$ signal

However, in the case of the selected driver (EL7158), where the minimum pulse width is 10ns, the design area does not exist for the $5 \mathrm{MHz}$ signal as it is shown in Figure 12. It implies that it is not possible to design the output filter with LC values that are feasible for the implementation because the curves intersect at very low capacitor values. Because of 
that, in this case, the LC filter is influenced only by the minimal driver pulse width and a modified control capable to cope with this problem is proposed and it will be explained in section III.

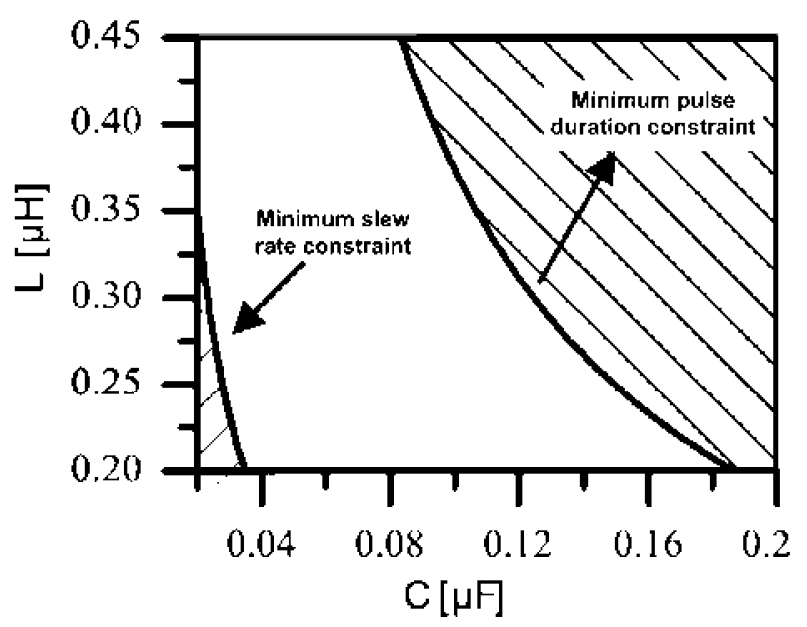

Fig. 12. LC design area in the case when the minimum driver pulse width is 10ns

\section{B. Switching frequency}

In the proposed multilevel converter, the multiphase buck converter operates at a pre-designed switching frequency, which needs to be carefully calculated at the system level, especially from the efficiency point of view. In the minimum time control calculation, the switching frequency $\left(f_{\mathrm{sW}}\right)$ also plays an important role. Although $\mathrm{f}_{\mathrm{sW}}$ does not appear directly in the calculation of $t_{\mathrm{ON}}$ and $\mathrm{t}_{\mathrm{OFF}}, \mathrm{f}_{\mathrm{sW}}$ is used to calculate $\Delta \mathrm{I}_{\mathrm{i}}$ that represents the change of the inductor current after a transition in the $\mathrm{i}^{\text {th }}$ phase and this is very important to calculate $\Delta \mathrm{t}, \mathrm{t}_{\mathrm{ON}, \mathrm{i}}$ and $\mathrm{t}_{\mathrm{OFF}, \mathrm{i}}$, as shown in equations 2,3 and 4 .

Figure 13 shows how the switching frequency affects the LC filter design area based on the aforementioned constraints (minimum driver pulse width is 1ns) in the case of the 8-phase phase buck converter that tracks a $5 \mathrm{MHz}$ RF signal. Although $\Delta \mathrm{I}_{\mathrm{i}}$ is different in each phase, it is always inversely proportional to $\mathrm{f}_{\mathrm{sw}}$. Therefore, with lower $\mathrm{f}_{\mathrm{sw}}$, higher $\Delta \mathrm{I}_{1}$ is obtained, which results in longer $\Delta \mathrm{t}$ due to higher $\sum_{i=1}^{N} \Delta I_{i}^{2}$ in equation 3. Apparently, a longer $\Delta \mathrm{t}$ with fixed voltage change means lower slew rate. It explains why the minimum slew rate constraint border moves to the left side when the $f_{\mathrm{sw}}$ decreases. 


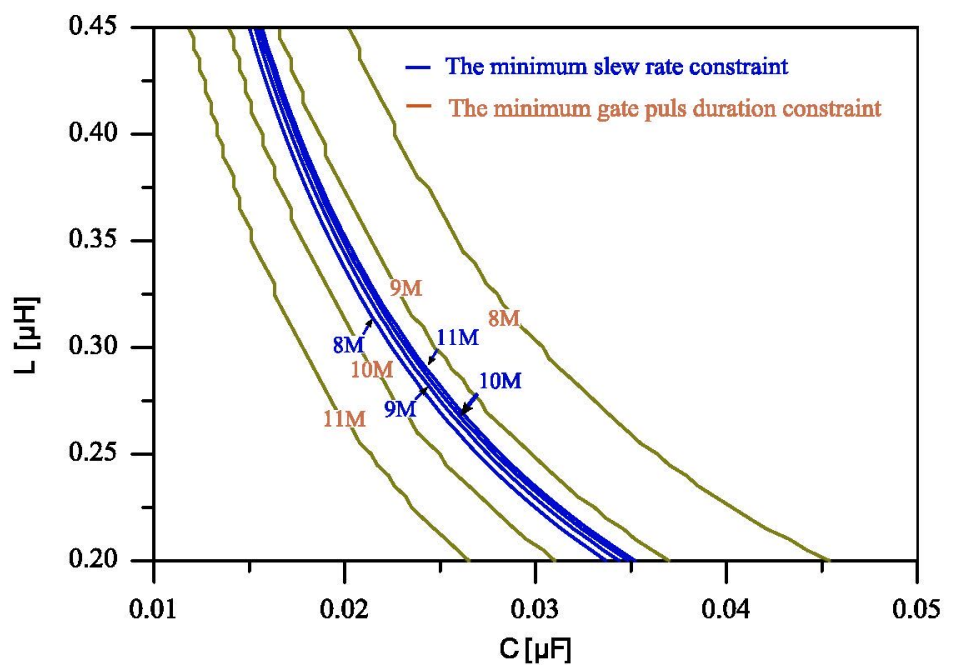

Fig. 13. LC design space for different switching frequencies

When a higher $\Delta \mathrm{I}_{\mathrm{i}}$ is substituted to calculate $\mathrm{t}_{\mathrm{ON}, \mathrm{i}}$ (during a step-up), the consequence is that $\mathrm{t}_{\mathrm{ON}}$ takes more percentage of $\Delta t$ (although $\Delta t$ also increases when $f_{\mathrm{sw}}$ decreases, $t_{\mathrm{ON}}$ is more sensitive to $f_{\mathrm{sw}}$ than $\Delta \mathrm{t}$ ). Similar conclusion is obtained for $\mathrm{t}_{\mathrm{OFF}}$ during the step down. In this situation, under the extreme cases explained in the previous subsection, the minimum pulse width is becoming shorter if $\mathbf{f}_{\mathrm{sW}}$ is increased. Therefore, it explains why the borderline obtained for the minimum voltage pulse duration moves to the right side when the $f_{\mathrm{sW}}$ decreases.

By analyzing Figure 13, it can be observed that when $\mathrm{f}_{\mathrm{sw}}$ is below $10 \mathrm{MHz}$, the $\mathrm{LC}$ filter design area is lost. Having in mind that for the wide bandwidth application the switching and gate power losses are usually the dominant ones in the case of the multilevel buck converter, the switching frequency should be selected as the one when the LC filter area starts to appear, i.e. when the design areas for the minimum voltage pulse duration constraint and the minimum slew rate constraint start to overlap.

In the case when it is impossible to find the design area for the LC filter due to the high minimum pulse width of the driver, like it is in the case of the $10 \mathrm{MHz}$ signal for example, the switching frequency should be selected having in mind the overall efficiency of the envelope amplifier using the improved control strategy presented in the following section.

\section{IMPROVED CONTROL STRATEGY}

In the proposed solution, digital control is used to control the multiphase buck converter. Besides the well-known advantages of digital control, such as smaller number of discrete components and less sensitivity to external influences, the complexity of the control in this application makes the digital control the only applicable option. Furthermore, the RF signal reference is implemented by digital signal processing techniques in RFPA system, which can be compatible with digital controller for the switching converter.

In order to implement a control for the envelope amplifier that can operate in real time, it is necessary to analyze the envelope reference inside a predefined time window. The controller can delay the envelope and analyze it inside a certain 
time interval and optimize the voltage transition of the multilevel converter during this time, i.e. decides when to make the voltage transition and what transition to make (one level or several).

\section{A. Transition shift for fast envelope}

Based on the constraints analysis of the proposed EA in the previous section, when the envelope has $10 \mathrm{MHz}$ of bandwidth, the minimum time control will have extremely small pulse widths during the transitions (the order of few ns, as it can be seen from Table I), in order to comply with the minimum slew rate. These time durations are very challenging for the transistor driver and, to our best knowledge, they exceed the limits of the commercially available offthe-shelf drivers. By applying the analysis explained in the previous section, it is impossible to find a design area for the output filter that would lead to realistic filter design using the minimum pulse duration time constraints of the EL7158 driver and the constraint regarding the minimum slew rate of the output signal in order to follow a $10 \mathrm{MHz}$ signal. To handle this extreme case, a special control strategy is needed.

In the normal control strategy, the transition is triggered when the envelope is higher than a predefined threshold voltage (step-up transition as example). Having in mind that the lower slew rate of the multiphase buck converter's transition comparing to the envelope slew rate would produce a distortion, this transition must be triggered in advance in order to keep converter's output voltage always higher than the envelope, as shown in Figure 14. It is possible to calculate the minimum time the transition trigger needs to shift because the envelope is known due to the aforementioned time delay. This problem can be resolved geometrically using the data from Figure 14. By assuming T1 is the transition before shifting, we can sweep the envelope above the $\mathrm{Tl}$ and find the point $\mathrm{M}$ that has the maximum difference between envelope and $\mathrm{T} 1\left(\mathrm{~d}_{\max }\right) . \mathrm{T} 2$ represents the transition after shifting, and $\mathrm{T} 2$ has to pass through $\mathrm{M}$ and be in parallel with T1. With $\mathrm{d}_{\max }$ and the slew rate of the transition $\left(\mathrm{SR}_{\mathrm{MTC}}\right)$, the minimum shifting time $\mathrm{t}_{\mathrm{ad}}$ can be obtained as:

$$
t_{a d}=\frac{d_{\max }}{S T_{M T C}}+\Delta t_{\text {margin }}
$$

A time margin is added to the shift time for the necessary back-off voltage if it is needed by the RF PA. This process takes up some computation time, so it is a potential problem for a real time implementation. Nevertheless, using this approach the proposed solution is operative beyond the two aforementioned constraints for fast envelope applications. 


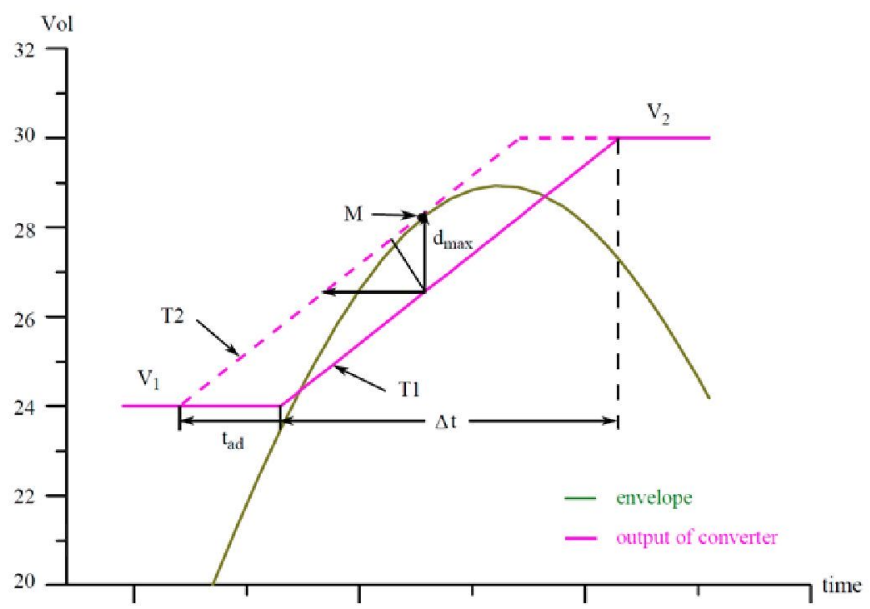

Fig. 14. Geomertrical representation of the proposed multilevel control for fast slew rates

\section{B. Transition synchronization and reorganization of phases}

As previously explained, sometimes it might be necessary to perform a voltage transition that goes across several voltage levels. These transitions are useful in the case when it is necessary to perform two or more consecutive simple voltage changes without long steady state between them. However, it might lead to more losses in the linear regulator, as the area between the converter's output voltage and envelope represents the losses of the linear regulator in the proposed EA.

In the model proposed in [31] it is illustrated that the voltage transition has to synchronize with the PWM signal of one phase (master phase). It means that the next transition can be triggered at least one switching period after the end of the previous transition. This penalizes the consecutive levels transition for fast envelope changing because one PWM cycle can be too long for certain slew rates. Taking advantage of the fixed phase shifting of a multiphase buck converter, any phase can be recognized as the master phase (first phase). Therefore, without changing the MTC model, the transition can be synchronized with any phase PWM signal. When the transition trigger comes, the phase whose beginning of PWM cycle is the closest to that moment must be selected as the master phase. This means that in a multiphase converter with $\mathrm{N}_{\text {phase }}$ phases a voltage transition can occur $\mathrm{T}_{\mathrm{PWM}} / \mathrm{N}_{\text {phase }}$ after the previous transition. Figure 15 shows simplified flow chart of the algorithm that selects the master phase after each voltage transition.

Phase reshuffle opens a possibility to perform fast consecutive jumps and in that way avoid additional power losses and high current peaks during a single transition across several voltage levels. Figure 16 shows time diagram of the output voltage, phase currents and PWM signals when the voltage transitions are always synchronized with the fixed master phase ( $1^{\text {st }}$ phase). In the same figure it is shown how the same transition would look like if the $8^{\text {th }}$ phase is selected as the master phase. It can be clearly observed how the distance between the consecutive voltage transitions is reduced. 


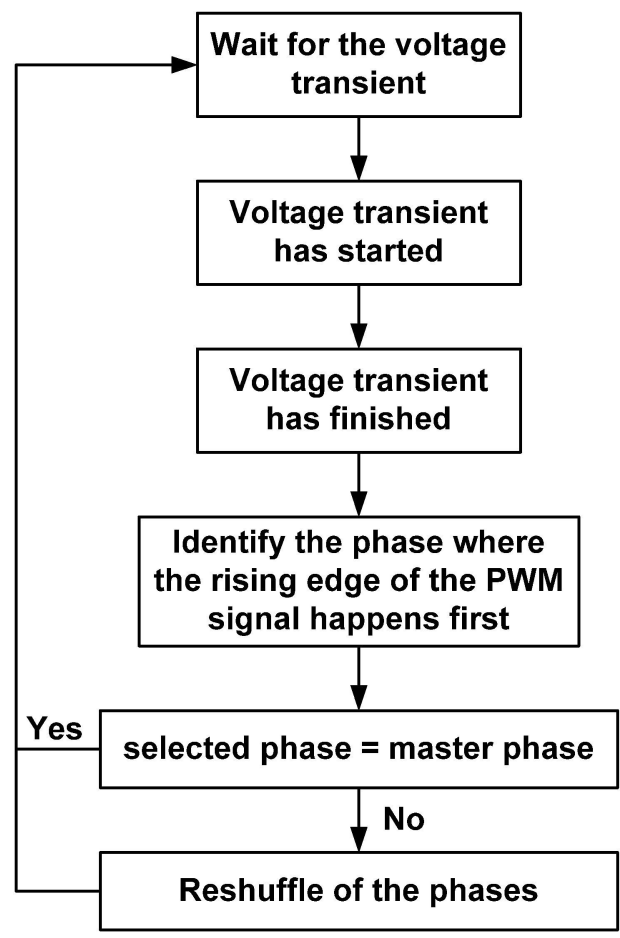

Fig. 15. Flow diagram for the phase reshuffle
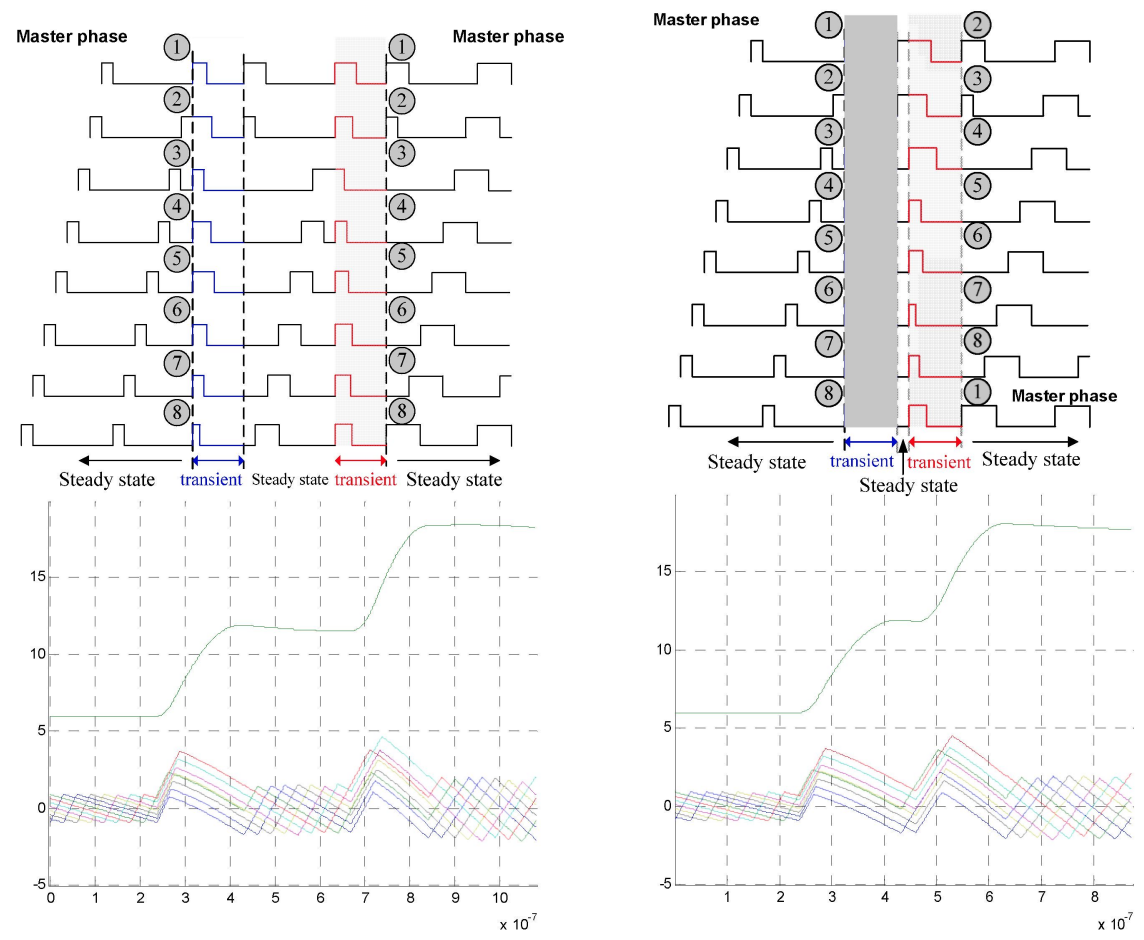

Fig. 16. Voltage transitions when the phases maintain its order (left) and when they are reshuffled (right)

IV. EFFICIENCY OPTIMIZATION

In order to maximize the efficiency of the complete envelope amplifier, the selection of the number of phases of the multilevel converter can be analyzed from two aspects:

- linear stage power loss 
- multiphase buck converter power loss

For the linear regulator, the losses are geometrically represented by the area between the converter's output voltage and envelope voltage and it is relatively easy to calculate it. For the multiphase buck converter, the losses estimation is more complicated. Having in mind that the multiphase converter will work at relatively high frequency (several MHz) parasitic source and loop inductances will have strong influence on the overall efficiency. Additionally, as it was previously explained, the converter operates with high RMS currents (high current ripple) in order to guarantee current sharing. Therefore, the conduction losses and turn off losses may be high. The power loss model for the multiphase buck converter was developed using models from the state of the art $[34,35]$. In the analysis, the static power consumption of the MOSFET drivers has been taken into account as well, because the consumption of the drivers and auxiliary supplies reaches several tens of $\mathrm{mA}$ and cannot be neglected.

It must be noticed that during the operation of the proposed envelope amplifier, the frequency of transition highly depends on the probability distribution of envelope. By analyzing in detail the waveform during the transition, the mechanism of how the transition increases the power loss can be understood. Figure 17 shows the phase current simulation in tracking of a 64QAM envelope with 4A load current. In the steady state all the phase currents can achieve ZVS for both transistors, because the minimum value is negative for each phase current. However, during the transitions there are phases which cannot achieve ZVS for the high side transistor, because the minimum current is not negative. This brings additional power losses. Moreover, because of the high peak values the RMS value is considerable and it increases the conduction loss in the transistors and filter inductance. The gate loss and the losses due to charging of the transistor's $\mathrm{C}_{\mathrm{oss}}$ are only slightly influenced by the transition. These additional power losses must be taken into account during the optimization of the topology.

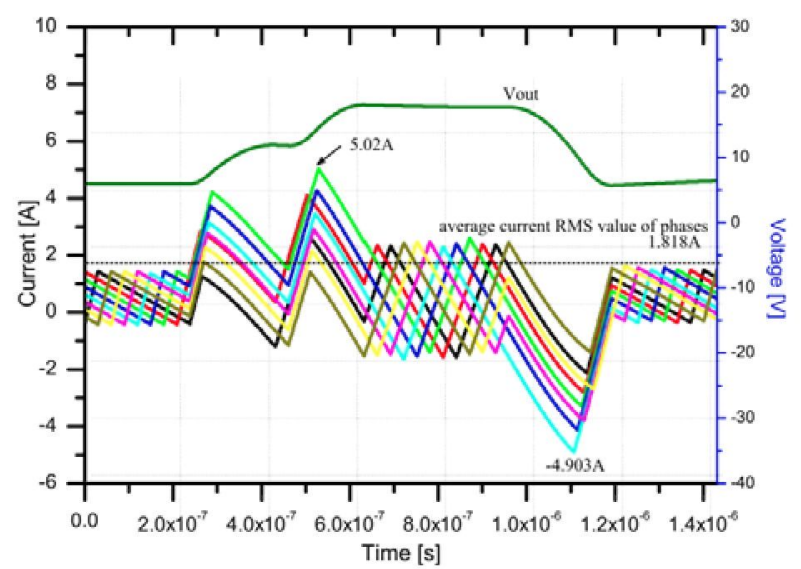

Fig. 17. A segment of current phase waveforms when the 8 phase buck converter is tracking an envelope in simulation

In the proposed multilevel converter, the number of available voltage levels is equal to the number of phases. With more phases, the multilevel converter has higher density of discrete output voltage levels, resulting in the feasibility of reducing the linear stage power loss. On the other hand, the increase in the number of phases also increases the static 
power loss of the multilevel converter. A trade-off is needed for the efficiency optimization. It has to be pointed out that the presented analysis is very general and more details on how to correctly estimate the power losses of an envelope amplifier using the information of the transmitted signal's probability distribution can be found in [15]. The main idea of this approach is to find the dependence of the envelope amplifier's power losses on the instantaneous value of the envelope, using, for example, power loss models in [34,35]. With this dependence defined as a function, $P_{\text {loss }}\left(V_{\text {envelope }}\right)$, it is relatively easy to calculate the average efficiency using the information regarding the envelope probability distribution:

$$
\begin{gathered}
P_{\text {average_out }}=\int_{0}^{V_{\text {envelope_max }}} \frac{v_{\text {envelope }}^{2}}{R_{\text {load }}} p\left(v_{\text {envelope }}\right) d v_{\text {envelope }} \\
P_{\text {average_power_loss }}=\int_{0}^{V_{\text {envelope_max }}} P_{\text {loss }}\left(v_{\text {envelope }}\right) p\left(v_{\text {envelope }}\right) d v_{\text {envelope }} \\
\eta=\frac{P_{\text {average_out }}}{P_{\text {average_out }}+P_{\text {average_power_loss }}}
\end{gathered}
$$

where $p\left(V_{\text {envelope }}\right)$ and $R_{\text {load }}$ are probability density function of the envelope and the effective load resistance seen by the envelope amplifier, respectively.

The influence of the number of phases on the power loss of the linear stage and the multilevel converter depends on the envelope, its probability distribution and the load. In the presented estimation, the envelope reference was taken from a 64QAM RF signal with bandwidths of $5 \mathrm{MHz}$ and $10 \mathrm{MHz}$, while the load of the envelope amplifier was a $10 \Omega$ resistor. The results are presented in Figure 18 showing that the multiphase converter composed of 4,8 and 16 phases has similar efficiency performance in the same load condition. The efficiency degrades for the 32-phase converter because the converter power loss significantly increases (due to the static power consumption), while the linear stage power loss does not exhibit so much improvement. For a 4 phase converter, the voltage difference between the consecutive levels is $12 \mathrm{~V}$ (in case of $V_{\mathbb{N}}=48 \mathrm{~V}$ ) resulting in a very high current peak during the transition, which presents a serious stress for the switching transistor. The power losses of the 4 phase converter are, approximately, $3 \mathrm{~W}$ lower than in the case of the 8 phase converter. However, due to lower number of generated levels, the power losses in the linear regulator are significantly higher (around $6 \mathrm{~W}$ ) which results in lower overall losses in the case of the 8 phase converter. Comparing 8 and 16 phase converters, 8 phase converter has slightly higher efficiency than the one with 16 phases. Additionally, since one of the main considerations in this work is the simplicity, 8 phases buck converter is used as the proposed envelope amplifier in the following sections. A summary of the 8 phase buck converter's power stage design, based on the complete previous analysis, is the following:

- $\quad$ The number of phases $\mathrm{N}_{\text {phase }}=8$

- The output filter: $\mathrm{L}=0.68 \mu \mathrm{H}, \mathrm{C}=0.3 \mu \mathrm{F}$

- The switching frequency $\mathrm{f}_{\mathrm{sw}}=4.2 \mathrm{MHz}$ 


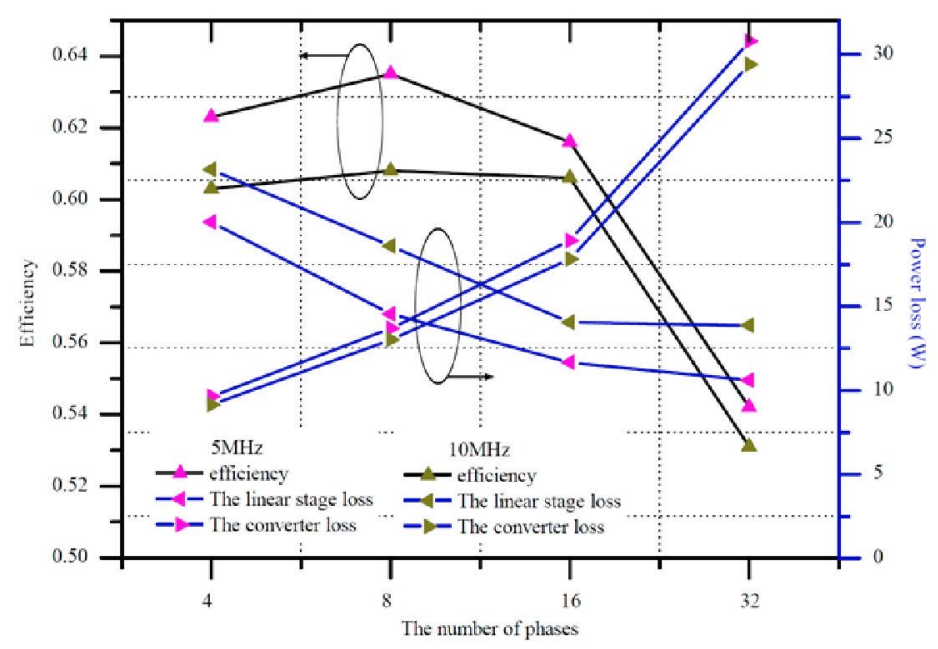

Fig. 18. The efficiency and power loss of the analyzed envelope ampliifer for different number of phases

The MOSFET that was used for the analysis presented in Figure 18 was FDS89141. Several transistors have been considered for the switching device and this transistor was selected as the best transistor in Si technology. Table II shows power loss estimation for the transistors in the case of a $10 \mathrm{MHz}$ signal without considering the power losses during the voltage changes. Power loss estimation using GaN EPC8009 shows significantly lower losses. However, due to the complexity of the 8 phase converter and GaN transistor package, the Si device was selected.

The filter inductor is implemented as an air core inductor in order to avoid core losses due to high switching frequency. The power loss breakdown is shown in Figure 19.

Table II Transistor power losses in the case of a 8 phase buck converter and a $5 \mathrm{MHz}$ signal

\begin{tabular}{|c|c|c|c|c|c|}
\cline { 2 - 6 } \multicolumn{1}{c|}{} & FDMC89521L & FDS3992 & BSC750N10ND & FDS89141 & EPC8009 \\
\hline Switching losses [W] & 3.77 & 2.85 & 3.66 & 2.97 & 1.58 \\
\hline Gate charge loss [W] & 2.695 & 2.04 & 2.69 & 1.02 & 0.14 \\
\hline Losses due to Coss [W] & 9.69 & 4.59 & 3.485 & 2.75 & 0.78 \\
\hline Conduction losses [W] & 0.47 & 2.07 & 2.3 & 2.7 & 3.97 \\
\hline Total losses [W] & 16.63 & 11.55 & 12.14 & 9.44 & 6.47 \\
\hline
\end{tabular}

\begin{tabular}{|c|c|c|c|c|c|}
\hline Ron $[\Omega]$ & 0.2 & 0.08 & 0.09 & 0.1 & 0.138 \\
\hline $\mathrm{Qg}[\mathrm{nC}]$ & 8 & 6 & 8 & 3 & 0.38 \\
\hline Cout $[\mathrm{pF}]$ & 250 & 118 & 90 & 65 & 20 \\
\hline
\end{tabular}

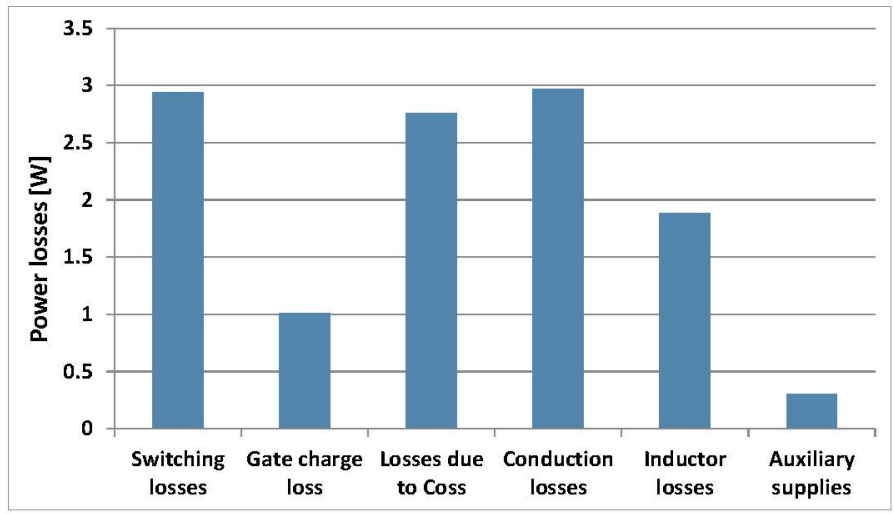

Fig. 19. Breakdown of the losses of the envelope amplifier based on a 8 phase buck converter in the case of a 5 MHz signal (FDS89141 transistor) 
The output filter value selection was performed complying only with the hardware limitation constraint. The reason for this is that in the case of 8 phases, there is no design area that can be found for the given objective application (5-10 MHz signal). Nevertheless, with the control strategy introduced in the previous section, the tracking performance of the converter can go beyond the minimum slew rate constraint that was already explained.

\section{V. $\quad$ EA EXPERIMENTAL RESULTS AND ANALYSIS}

After the analysis that has been presented in the previous sections, a prototype with the following specifications was built:

- Input voltage: $48 \mathrm{~V}$

- Peak power of $170 \mathrm{~W}$

- Output voltage range: $0 \mathrm{~V}$ to $42 \mathrm{~V}$

- Bandwidth of the tracked RF signal: $5 \mathrm{MHz}-10 \mathrm{MHz}$

An 8-phase buck converter was built based on the earlier explained efficiency optimization and considering the complexity of the proposed envelope amplifier. The details of the built multiphase buck converter are the following:

- High/low side MOSFET: FDS89141

- $\quad$ MOSFET driver: EL 7158

- Digital controller: FPGA Virtex-5

- Output inductor: 143-11J12L Coilcraft $(680 \mathrm{nH})$

- $\quad$ Output capacitor: $0.3 \mu \mathrm{F}$

The MOSFETs have been selected optimizing the efficiency of the multilevel converter as explained in the previous section. Because of the high switching frequency, two independent drivers were used for high and low side MOSFETs, instead of the classical solution with a bootstrap driver. Similar to the control of a 4-phase buck converter in [31], an FPGA was used as the controller of the 8-phase buck converter. Due to the higher bandwidth envelope specification, smaller transition time is needed resulting in higher digital control resolution requirement. An FPGA Virtex-5 from Xilinx family with $200 \mathrm{MHz}$ clock frequency (5ns resolution) was selected as the controller. Since more complex control strategies including transition shift and transition synchronization introduced in previous sections are implemented, this control employed an embedded microprocessor in the design.

The linear regulator is the final stage of the proposed envelope amplifier for EER technique, although it is not necessary for ET. For its design, the selection of the transistor and operational amplifier is of crucial importance for the wide bandwidth. The operational amplifier needs to be fast and able to handle higher voltage than output voltage of the linear regulator. In this implementation of the EA, the PA107 from APEX Microtechnology is used as the solution for the high speed linear regulator. It is actually a high voltage and high bandwidth power amplifier with the auxiliary power 
supply separated from the drain of main power transistor. This amplifier can reproduce slew rates up to $3 \mathrm{kV} / \mu \mathrm{s}$ and this feature makes it suitable candidate for this application.

\section{A. Experimental results}

The static efficiency measurement of the implemented 8-phase buck converter is shown in Figure 20. The EA is optimized and built for relatively high currents ( $2 \mathrm{~A}-4 \mathrm{~A}$ ) and the measured efficiency is between $85 \%$ and $95 \%$ when it supplies these currents. The efficiency drops down significantly mostly at light load due to static power losses, because it is necessary to supply 16 drivers and 16 isolation chips and this power consumption is very important at light load.

After the static efficiency measurements, the efficiency of the complete EA is measured in the case of a $5 \mathrm{MHz} R \mathrm{RF}$ signal. Figure 21 shows the measured waveforms of the envelope amplifier in this case. The average power that was supplied was $39.4 \mathrm{~W}$, while the peak power was around $176.4 \mathrm{~W}$. The measured overall efficiency was $61.3 \%$.

It can be observed that the multilevel converter is able to follow the envelope, while the voltage reproduced by PA107 is not identical to the envelope reference. This will have significant influence on the linearity of the EER transmitter as it is explained and measured in section VI.

The implemented multilevel converter is able to follow even faster envelopes using the earlier explained phase reshuffle and transition shift. Figure 22 shows the multilevel voltage waveform in the case of a $10 \mathrm{MHz} 64 \mathrm{QAM}$ RF signal envelope. This test is carried out without using a linear regulator, because the linear amplifier PA107 is not able to follow such fast envelopes. The load was a $10 \Omega$ resistor. The average power that was supplied was $88 \mathrm{~W}$, while the peak power was $176 \mathrm{~W}$. The average power is higher than in the previous case because there is no linear amplifier to correctly shape the envelope. The measured efficiency was $88 \%$

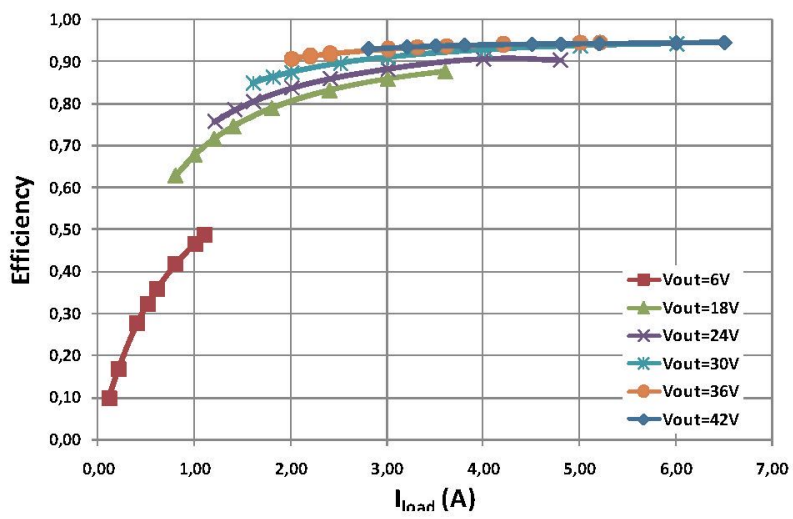

Fig. 20. Steady state efficiency measurementss of the implemented multiphase converter 


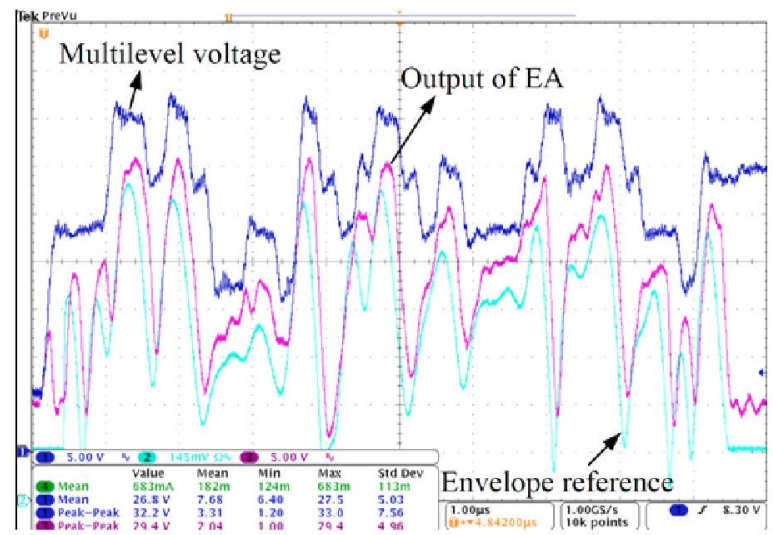

Fig. 21. Measured waveforms of the implemented envelope amplifier tracking a $5 \mathrm{MHz} 64 \mathrm{QAM}$ signals

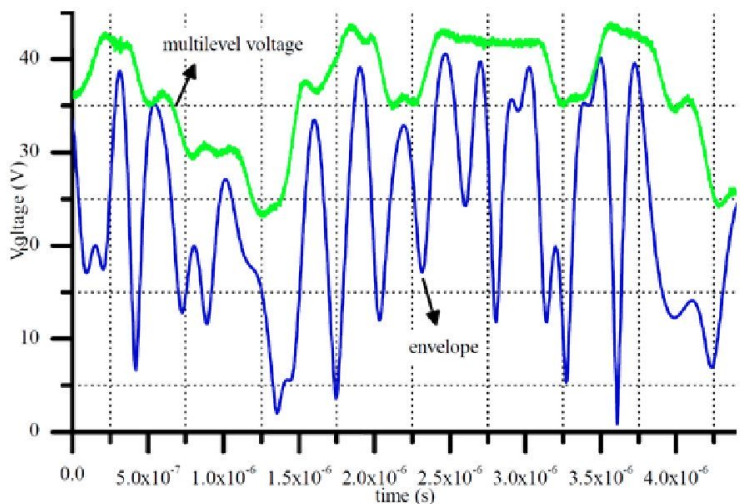

Fig. 22. Measured output voltage of the implemented 8 phase buck converter tracking a $10 \mathrm{MHz} 64 \mathrm{QAM}$ signal

\section{B. Sensitivity analysis of the multilevel converter}

It can be observed from the measured waveform of the multilevel converter in Figures 21 and 22 that the oscillation and overshoot usually appear after the transition. There are several causes for this unwanted waveform, which are the main discussions in this section.

The MTC model approximation: In the minimum time control model presented in [31] it is assumed that the output voltage is changed linearly during the transition. However, when the interval times ( $\mathrm{t}_{\mathrm{ON}, \mathrm{i}}$ and $\left.\mathrm{t}_{\mathrm{OFF}, \mathrm{i}}\right)$ based on the proposed MTC model are applied to the converter, the output voltage exhibits different shape from the linear change assumption, as shown in Figure 23. The simulation shows that the output voltage of quasi-quadratic curve is divided by two frames. In the first frame, the curve starts to increase and the slope of the curve also increases because the current injecting into the output capacitor is increasing by turning on all the phases (high side switches). Then, after all the phases switch off, the current injecting into the output capacitor starts to decrease. The result is that the output voltage keeps increasing, but with a decreasing slope and it saturates at the end of transition. The knee point of the output voltage curve is also the intersection point of the output voltage curve and the linear output voltage assumption. In the moment when the transition should be ended, the output voltage is lower than the target value.

In order to explain how the deviation of the output voltage from the linear approximation influences the minimum time control, the transition in Figure 23 is used as an example for the analysis. Before all phases switch off, the output is 
very close to the linear assumption. After that, the output is obviously higher than the assumed one, so that the actual voltage on the inductors is higher than the assumed one during the switch off interval This results in higher slope of ramping down current and lower current level at the end of transition than in the calculation. The sum of the areas under the phase currents during the transition that represents the real charge increment, $\Delta \mathrm{Q}_{\text {real, }}$ will be smaller than the one calculated by the linear voltage approximation $\left(\Delta \mathrm{Q}_{\text {need }}\right)$. Therefore, the voltage at the end of transition does not reach the expected level and the oscillations after the transition are observed. This phenomenon is not important in the case of a high $\Delta \mathrm{Q}_{\text {real }}$ related to large transition time, because $\left(\Delta \mathrm{Q}_{\mathrm{real}}-\Delta \mathrm{Q}_{\mathrm{need}}\right) / \Delta \mathrm{Q}_{\text {real }}$ caused by the linear assumption is small. In contrast, the influence is significant in the case of low $\Delta \mathrm{Q}_{\text {real }}$ for a fast transition.

On the other hand, it is possible to use a quasi-quadratic curve instead of a linear assumption to improve the MTC model. However it will make the calculation of $\mathrm{t}_{\mathrm{ON}, \mathrm{i}}$ and $\mathrm{t}_{\mathrm{OFF}, \mathrm{i}}$ very complicated. In this work, a calibration in simulation by adjusting $\mathrm{t}_{\mathrm{ON}, \mathrm{i}}$ and $\mathrm{t}_{\mathrm{OFF}, \mathrm{i}}$ is performed in order to approach $\Delta \mathrm{Q}_{\text {need. }}$. Once these parameters of MTC are identified, they will be a constant value for specific prototype regardless of the load condition as it is explained in detail in [31].

The digital control resolution: Comparing to the analog control, the resolution of a digital control is always a drawback. However, it is nearly impossible to apply analog control to MTC of multiphase converter due to the complexity. As aforementioned, $5 \mathrm{~ns}$ clock period is used in this work. This resolution is sufficient when the transition time is long ( $>1 \mu \mathrm{s})$, but it can be an issue when the transition time is short (200ns in this case) due to fast tracking requirement. The maximum error of each pulse due to digital control resolution (5ns) is $2.5 \mathrm{~ns}$. When the transition time enters the range of $200 \mathrm{~ns}$, for 8-phases buck converter, the minimum interval $\mathrm{t}_{\mathrm{ON}}$ or $\mathrm{t}_{\mathrm{OFF}}$ can be around $20 \mathrm{~ns}$.

The dead-time influence: Like in the case of the digital control resolution, the dead-time is usually negligible when the transition time is long. When they are comparable, the dead-time becomes an issue in MTC. Figure 24 shows waveforms of one phase in the step-up transition. When one phase enters the transition, the low side switch $\left(\mathrm{L}_{\mathrm{s}}\right)$ at first turns off at $\mathfrak{t}_{1}$. During the dead-time $\mathbf{t}_{\text {deadl }}$, the inductor current charges the node capacitor resulting in the voltage across inductor increasing from negative to positive. At first, the phase current keeps decreasing with the decreasing slope until the voltage across the inductor and the phase current slope are zero. Afterwards, it starts increasing with increasing slope. At $t_{2}$, the high side switch turns on and the dead-time ends. In the dead-time, the sum of all phase currents is still in steady state, so the actual minimum time control starts at $\mathrm{t}_{2}$. During the dead-time $\mathrm{t}_{\text {dead2 }}$, the inductor current starts discharging the node capacitor when the high side switch turns off at $\mathrm{t}_{3}$, and then with the node voltage varying the phase current will reach the peak and go down afterwards. Therefore, the actual end point of $t_{\mathrm{ON}, \mathrm{i}}$ is the peak current point between $t_{3}$ and $t_{4}$. When the control quits MTC, the converter actually enters the steady state at $t_{5}$ when the low side 
switch turns off. From Figure 24, it is observed that a dead-time $\mathbf{t}_{\text {deadl }}$ should be subtracted from the transition time $\Delta \mathrm{t}$. Having this in mind the pre-calculated times have to be adjusted as follows:

$$
\begin{gathered}
t_{\text {on }}=t_{\text {on_theory }}+0.5\left(t_{\text {dead } 1}+t_{\text {dead } 2}\right) \\
t_{\text {off }}=t_{\text {off_theory }}-0.5\left(t_{\text {dead } 1}+t_{\text {dead } 2}\right)
\end{gathered}
$$

where $t_{\text {on_theory }}$ and $t_{\text {off_theory }}$ are theoretically calculated ON and OFF times, while $t_{\text {dead } 1}$ and $t_{\text {dead } 2}$ are the dead times between the high side and low side gate pulse.

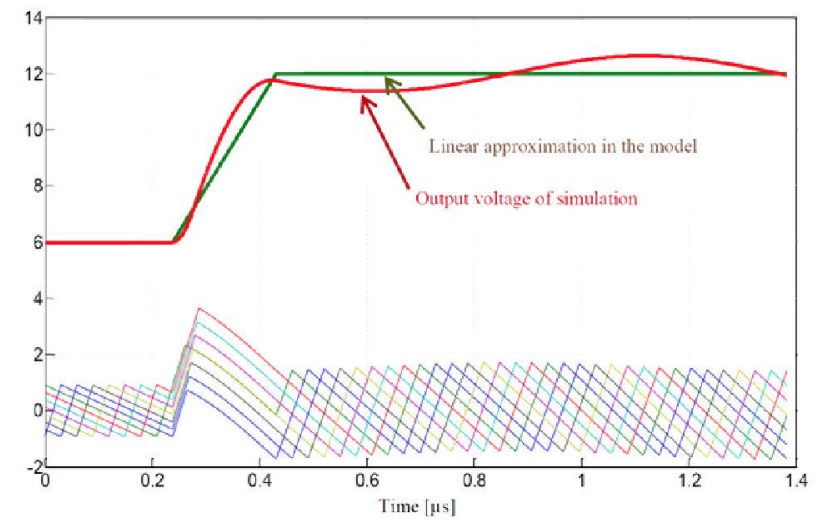

Fig. 23. The output voltage of the 8 phase buck conerter in the MTC model and in simulation

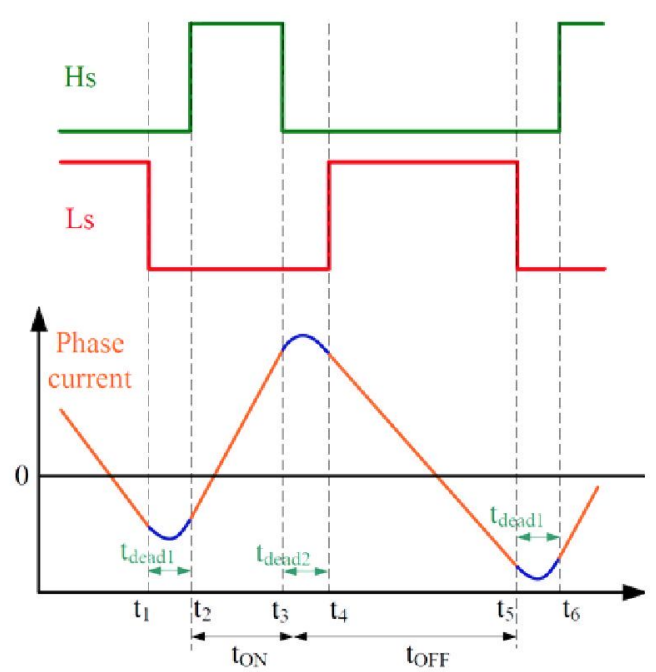

Fig. 24. Gate pulses and a phase current during the minimum time control including the dead-time

The capacitance variation with the voltage bias: In this envelope amplifier, the output of multiphase buck converter varies from low voltage $(6 \mathrm{~V})$ to relatively high voltage $(42 \mathrm{~V})$. Depending on the technology, the output capacitance may not be constant within this range of a voltage bias. With DC bias, the capacitance might have a decrease up to $20 \%$ from its nominal value and it can influence the calculation of MTC model. In order to include this characteristic into the model, it is possible to utilize a polynomial fitting curve to model the nonlinear capacitance.

The inductance mismatch: More than 10\% tolerance of inductance is typical for commercial inductors, which is not taken into account in the MTC model. In order to know how sensitive the transition performance is to the inductance mismatch, the measured inductances were substituted into the model. The measured inductances had a $5 \%$ tolerance. The 
simulations that we have performed showed a slight oscillation that is nearly negligible indicating that the voltage transition is not sensitive to the inductance tolerance.

Figure 25 summarize a transition performance (from $6 \mathrm{~V}$ to $12 \mathrm{~V}$ ) in both the simulation and the measurement. When all the aforementioned details are taken into account (dead time, nonlinear voltage change, etc.) the improvement in the transient response is obvious. Nevertheless, the influence of the digital control resolution and the inductor mismatch always exists and there is always a small oscillation after the transient.
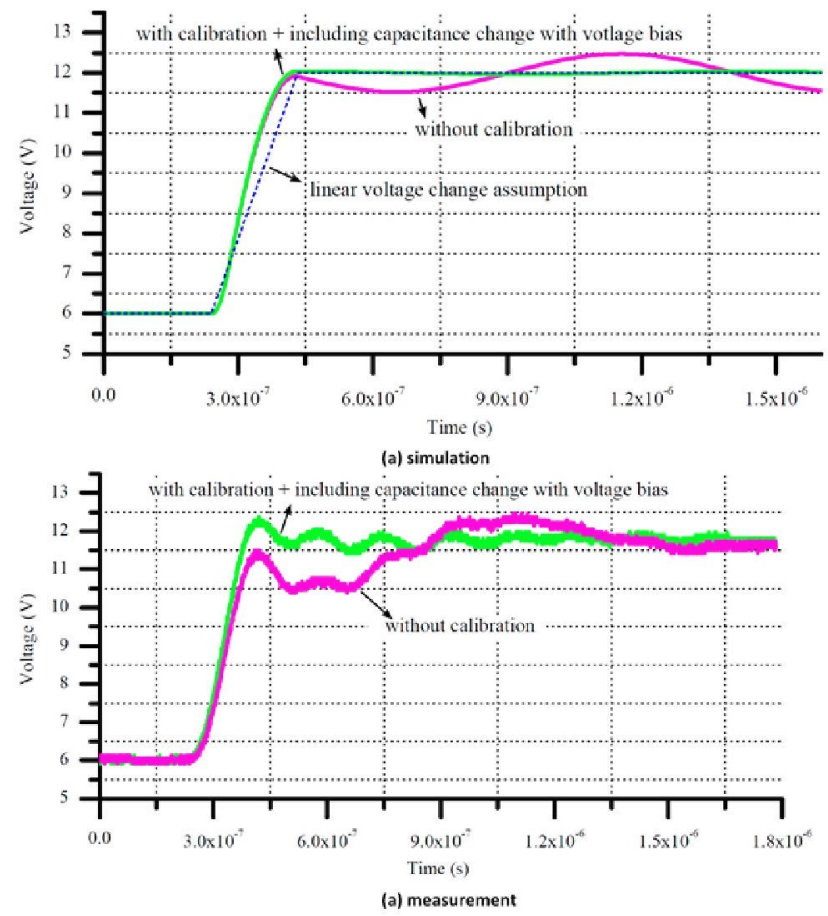

Fig. 25. The transition waveform with and without calibration

\section{EER POWER AMPLIFIER MEASUREMENTS}

In order to validate the concept of the power saving with the proposed envelope amplifier, an EER power amplifier system with a Class F power amplifier has been implemented. For the RFPA, a high efficiency switch-model Class F power amplifier that uses GaN transistor is employed. It is a commercial device RF3931 from RF Micro Devices using GaN-based technology. The performance of a power amplifier is usually limited by the transistor drain-source capacitor, $\mathrm{C}_{\mathrm{ds}}$ and conduction resistance, $\mathrm{R}_{\mathrm{on}}$. The $\mathrm{C}_{\mathrm{ds}}$ makes it difficult to control the harmonics of the load network. A device with small $\mathrm{C}_{\mathrm{ds}}$ can reach high operating frequency and help generate high order harmonics. GaN-HEMT transistors are good candidates for Class $\mathrm{F}$ amplifiers because, not only they have low $\mathrm{C}_{\mathrm{ds}}$ and $\mathrm{R}_{\text {on }}$ that is favorable for improving efficiency, but also they have shown significantly higher power density than GaAs and silicon devices [36]. Additionally, they have higher breakdown voltage and lower drain impedance than LDMOS. The maximum drain efficiency of $67.3 \%$ is obtained at the output power of $39.5 \mathrm{~W}$, Figure 26 . It can also be seen that for the majority of the measured frequencies the drain 
efficiency is higher than $60 \%$. The schematic and the photograph of the transmitter are shown in Figure 27 . The envelope amplifier is composed of the 8 phase buck converter and a linear amplifier (PA107) in series.

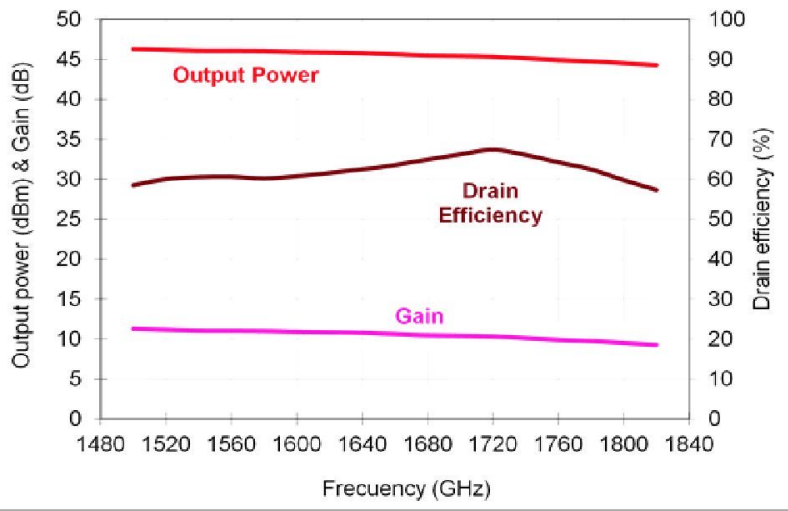

Fig. 26. Drain efficiency, Gain and Output power of the implemented class $\mathrm{F}$ amplifier dependence on different carrier frequencies

The test conditions are the following:

- $\mathrm{V}_{\mathrm{IN}}=48 \mathrm{~V}$

- $\mathrm{V}_{\text {EAmax }}=42 \mathrm{~V}$

- $\mathrm{f}_{\mathrm{SW}}=4.2 \mathrm{MHz}$

- $\mathrm{f}_{\text {carrier }}=1.64 \mathrm{GHz}$

- modulation is a 64QAM

- envelope bandwidth is up to $5 \mathrm{MHz}$

where $V_{I N}$ is the input voltage of multiphase buck converter, $V_{\text {EAmax }}$ is the maximum output voltage of both multiphase converter and linear amplifier; $f_{S W}$ is the buck converter switching frequency and the $f_{\text {carrier }}$ is the carrier frequency of the signal that is the input of Class $\mathrm{F}$ amplifier.

The EER system prototype is integrated by removing the bias filter of Class $\mathrm{F}$ amplifier and connecting the output of PA107 and the supply input of Class F amplifier as shown in Figure 27, using the load resistance of $50 \Omega$.

In the test bench, the control signals of the multiphase buck converter are provided by an FPGA Virtex-5 in which the envelope information is also stored. The envelope reference of linear amplifier PA107 is given by a signal generator 81150A from Agilent with the look-up data generated by MATLAB. The phase modulation is also generated by MATLAB and stored in a vector signal generator E4438C also from Agilent that generates the $1.64 \mathrm{GHz}$ carrier.

There are two challenges to carry out the EER system measurement. One is to synchronize all the signal generators, oscilloscope, and also the FPGA by giving them a reference clock. The other is to align the phase modulation and the amplitude modulation. It is not possible to align them visually when the high carrier frequency is used, particularly for an EER system. In order to accomplish it, phase modulation is fixed, while the envelope is delayed, until a sufficient level of linearity is obtained. 
Figure 28 shows a segment of EER power amplifier measurement. As it is designed, the output of multiphase buck converter is instantaneously changing the level to track the envelope and it always stays at a higher level than the envelope to avoid amplitude distortion. In the wide bandwidth case (5 MHz signal) which is shown in Figure 28, the envelope is varying very fast and in order to follow it, the multiphase buck converter skips some transition levels making voltage jumps across two voltage levels. It is important to notice that the bandwidth of the tracked envelope is higher than the switching frequency of the multiphase converter. Clearly, the reason for this is the non linear control which is employed. In the theoretical discussion presented in [28] in the case of linear control and an ideal low pass filter, the minimum ratio between the converter's large signal bandwidth and its switching frequency is one.

The Adjacent Channel Power Ration measurement of the RF output signal was performed and the results are summarized in Figure 29 for different signal bandwidths. The practical way to measure the ACPR is to measure the power in a frequency window adjoining the signal channel from the low frequency side with the width equal to the signal bandwidth. In this way, we obtain ACPR_L. In the same way, but measuring the window from high frequency side, the ACPR_H is measured. It can be seen that the linearity performance degrades with higher bandwidth RF signal. This is caused by the large signal limitation of the linear amplifier which needs to have large signal bandwidth (around 3 times higher than the signal bandwidth). In addition, these ACPR performances can be significantly improved by applying predistortion techniques.

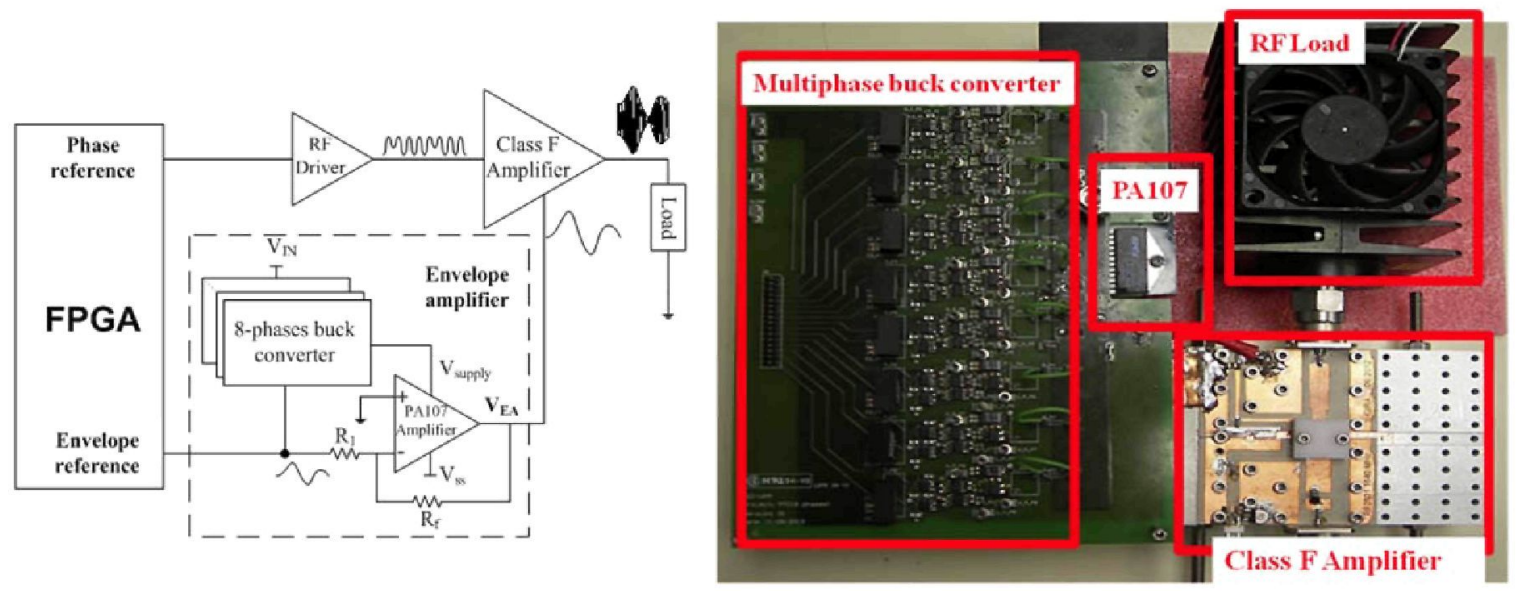

Fig. 27. Simplified bock schematic of the implemented EER transmitter and a photograph of the setup 


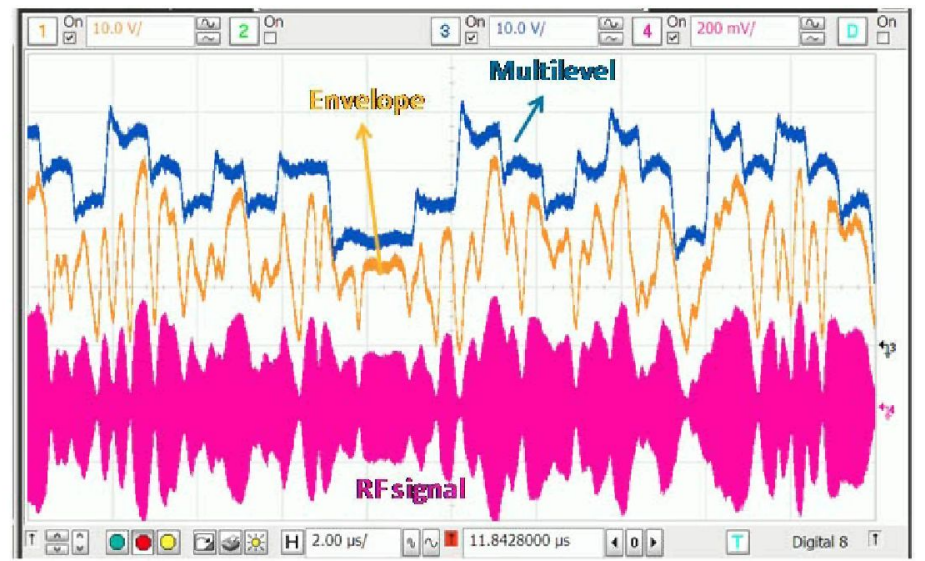

Fig. 28. Waveforms of the multiphase buck converter output, envelope amplifier output and EER transmitter output for a 5MHz 64QAM signal

The efficiency of the implemented EER power amplifier is measured with constant voltage and the proposed multilevel envelope amplifier and the results are shown in Figure 30 for different signal bandwidths. The power savings by employing the proposed multilevel envelope amplifier are also shown in the same figure. The average output power is $6.95 \mathrm{~W}$ for all the cases. At low bandwidths, when the multilevel converter follows the signal envelope closely, the drain efficiency can be boosted from $21.6 \%$ to $30.1 \%$. In this case, the power savings are around $9 \mathrm{~W}$. In the case of a $5 \mathrm{MHz}$ signal the average efficiency of the implemented amplifier is $25 \%$. This results in $4 \mathrm{~W}$ of power savings comparing it with the case when the RF amplifier is supplied with constant voltage supply, i.e. the power losses are reduced for $15 \%$. It has to be noticed that the 8-phase buck converter is optimized at higher output power. Therefore, high system efficiency and more power saving can be expected for high power EER power amplifier. The fact that the efficiency decreases with the wider bandwidth signal matches the expectation, since the multilevel voltage is not able to track the envelope as fast as the low bandwidth signal. This results in higher loss in the linear amplifier.

The class $\mathrm{F}$ amplifier presents an impedance of approximately $120 \Omega$ to the envelope amplifier. Due to this, the designed multiphase converter operates in a zone of relatively poor efficiency, well below $80 \%$. If the presented EER transmitter (RF amplifier together with the envelope amplifier) were optimized, the presented efficiency numbers would be significantly higher. Nevertheless, the presented efficiency results clearly show the impact of the presented topology on the overall power losses. 


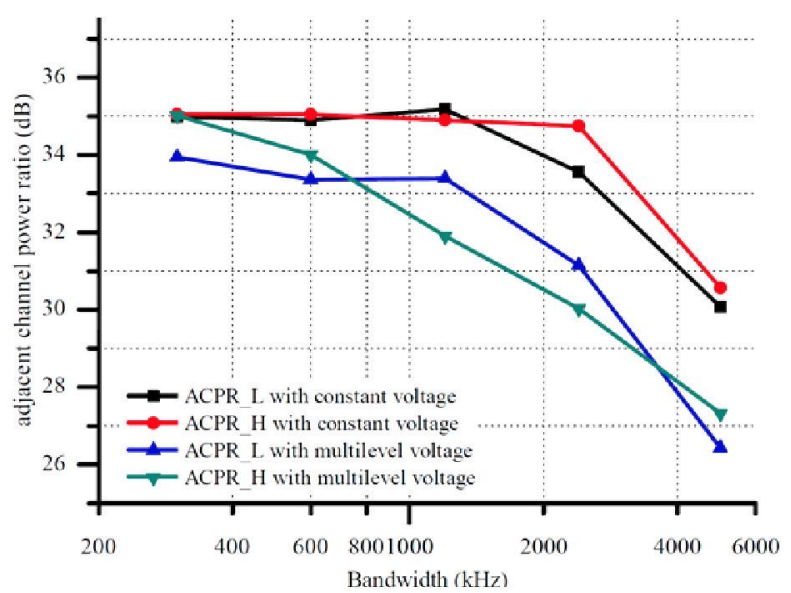

Fig. 29. The summary of ACPR of the implemented EER transmitter for different signal bandwidths

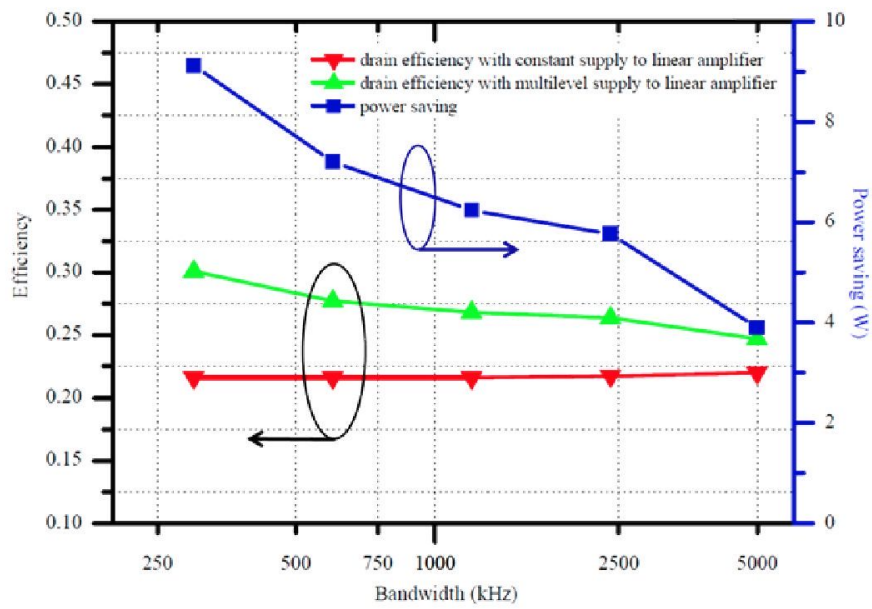

Fig. 30. The efficiency improvement and power saving of EER transmitter due to the multilevel voltage supply

\section{CONCLUSIONS}

The presented work shows one possible implementation of a multilevel converter that acts as the envelope amplifier in ET and EER. The proposed multilevel converter consists of only one power stage, a multiphase buck converter, which is less complex for the hardware implementation comparing to the state of the art solutions that are normally two power stage topologies. Nevertheless, control is significantly more complex because it is necessary to implement minimum time transients and to properly design the output filter and a switching frequency for the given transmitted signal. The presented analysis showed the existence of a precisely defined area for the output filter design. The number of phases, desired slew-rates, driver speed and switching frequency have huge impact on the size and existence of this design area. The presented analysis has shown that for wide bandwidth signals the driver's capability to produce small pulses is of the outmost importance. For the tested wide bandwidth RF signals it was impossible to obtain the output filter design area due to the transistor driver limitations. Due to this problem, a modified control based on transition shifts and phase reshuffle was proposed since it may help to track fast slew rate signals even in the cases when the output filter design 
area is not obtained. The proposed transition shift decreases the envelope amplifier efficiency as the multilevel converter does not follow the RF envelope ideally.

By using the proposed deign methodology, the number of phases of the multiphase converter can be optimized in order to obtain the highest possible efficiency. In the case of an RF signal that has 5 to $10 \mathrm{MHz}$ of bandwidth, the optimal number of phases is 8 and it is possible to select switching frequency lower than the bandwidth of the tracked signal. In this work, the switching frequency of $4.2 \mathrm{MHz}$ was used.

The sensitivity of the design is analyzed as well. It has been shown that the design is very sensitive to the time resolution that is used for the control of the minimum time transient. Other parameters, such as dead-times or inductance mismatch, can be mitigated or do not influence the quality of the converter's dynamic response significantly.

In order to validate the concept, an 8 phase buck converter has been built. It has been shown that it is possible to track signals with bandwidths as high as $10 \mathrm{MHz}$, using significantly lower switching frequency than in solutions based on PWM. The implemented multiphase converter has been integrated with a nonlinear class F amplifier in order to test its performance in an EER application. The efficiency measurements demonstrated that the multilevel converter decreases power losses for approximately $15 \%$ comparing to a constant power supply in the case of a $5 \mathrm{MHz}$ signal. In the case of signals width lower bandwidth, the power loss reduction is significantly higher since the multilevel converter follows the $\mathrm{RF}$ envelope almost ideally.

The spectrum measurements showed that ACPR is higher than $30 \mathrm{~dB}$ for signals with bandwidths up to $5 \mathrm{MHz}$. By applying predistortion, ACPR could be significantly improved.

\section{REFERENCES}

[1] "AN10923-1.5GHz Doherty power amplifier for base station applications using the BLF6G15L-250PBRN", NXP, Application Note, Rev.1., 14. March 2011

[2] D.J. Perreault, "A New Power Combining and Outphasing Modulation System for High-Efficiency Power Amplifiaction", IEEE Trans. on Circuits and Systems I,Vol.58, Issue 8, August 2011

[3] M.C.W.Hoyerby, M.A.E.Andersen, "Ultrafast tracking power supply with fourth-order output filter and fixed-frequency hysteretic control", IEEE Transactions on Power Electronics, Vol.23, No.5, Sep. 2008, pp. 2387-2398

[4] P.G. Blanken, R. Karadi, H.J. Bergveld, "A 50MHz Bandwidth Multi-Mode PA Supply Modulator for GSM, EDGE and UMTS Application", IEEE Radio Frequency Integrated Circuits Symposium, RFIC 2008, 15-17 June 2008

[5] P. Midya, K. Haddad, and M. Miller, "Buck or boost tracking power converter," Power Electronics Letters, IEEE, vol. 2,no. 4, pp. 131-134, Dec 2004.

[6] A. Soto, J. Oliver, J. Cobos, J. Cezon, and F. Arevalo, "Power supply for a radio transmitter with modulated supply voltage," in Applied Power Electronics Conference and Exposition, 2004. APEC '04. Nineteenth Annual IEEE, vol. 1, 2004, pp. 392-398 Vol.1.

[7] V. Pinon, F. Hasbani, A. Giry, D. Pache, and C. Garnier, "A single-chip WCDMA envelope reconstruction LDMOS PA with 130MHz switched-mode power supply," in Solid-State Circuits Conference, 2008. ISSCC 2008. Digest of Technical Papers. IEEE International, Feb 2008, pp. 564-636.

[8] M. Bathily, B. Allard, and F. Hasbani, "A 200-MHz integrated buck converter with resonant gate drivers for an RF power amplifier," Power Electronics, IEEE Transactions on, vol. 27, no. 2, pp. 610-613, Feb 2012.

[9] Eberle, W.; Zhiliang Zhang; Yan-Fei Liu; Sen, P.C., "A Practical Switching Loss Model for Buck Voltage Regulators," Power Electronics, IEEE Transactions on, vol.24, no.3, pp.700,713, March 2009

[10] G. B. Yundt, "Series- or parallel-connected composite amplifiers," Power Electronics, IEEE Transactions on, vol. PE-1, no. 1, pp. 48-54, Jan 1986. 
[11] H. Ertl, J. Kolar, and F. C. Zach, "Basic considerations and topologies of switched-mode assisted linear power amplifiers," Industrial Electronics, IEEE Transactions on, vol. 44, no. 1, pp. 116-123, Feb 1997.

[12] P. Midya, "Linear switcher combination with novel feedback", in Power Electronics Specialists Conference, 2000. PESC 00. 2000 IEEE 31st Annual, vol. 3, 2000, pp. 1425-1429 vol.3.

[13] Jin, Q.; Ruan, X.; Ren, X.; Xi, H., "High Efficiency Switch-Linear Hybrid Envelope-Tracking Power Supply with Step-Wave Approach," Industrial Electronics, IEEE Transactions on , March 2015

[14] V. Yousefzadeh, E. Alarcon, and D. Maksimovic, "Band separation and efficiency optimization in linear-assisted switching power amplifiers," in Power Electronics Specialists Conference, 2006. PESC '06. 37th IEEE, June 2006, pp. 1-7.

[15] Vasic, M.; Garcia, O.; Oliver, J.A.; Alou, P.; Cobos, J.A., "Theoretical Efficiency Limits of a Serial and Parallel Linear-Assisted Switching Converter as an Envelope Amplifier," Power Electronics, IEEE Transactions on , vol.29, no.2, pp.719,728, Feb. 2014

[16] C. Hsia, A. Zhu, J. Yan, P. Draxler, D. Kimball, S. Lanfranco, and P. Asbeck, "Digitally assisted dual-switch high efficiency envelope amplifier for envelope-tracking base-station power amplifiers," Microwave Theory and Techniques, IEEE Transactions on, vol. 59, no. 11, pp. 2943-2952, Nov 2011.

[17] Sankman, J.; Song, M; Ma, D., "Switching-Converter-Only Multiphase Envelope Modulator with Slew Rate Enhancer for LTE Power Amplifier Applications," Power Electronics, IEEE Transactions on, February 2015

[18] J. Staudinger, B. Gilsdorf, D. Newman, G. Norris, G. Sadowniczak, R. Sherman, and T. Quach, "High efficiency CDMA RF power amplifier using dynamic envelope tracking technique," in Microwave Symposium Digest. 2000 IEEE MTT-S International, vol. 2, June 2000 , pp. $873-876$ vol.2.

[19] B. Sahu and G. Rincon-Mora, "A high-efficiency linear rf power amplifier with a power-tracking dynamically adaptive buckboost supply," Microwave Theory and Techniques, IEEE Transactions on, vol. 52, no. 1, pp. 112-120, Jan 2004.

[20] J. Jeong, D. Kimball, M. Kwak, C. Hsia, P. Draxler, and P. Asbeck, "Wideband envelope tracking power amplifier with reduced bandwidth power supply waveform," in Microwave Symposium Digest, 2009. MTT '09. IEEE MTT-S International, June 2009, pp. 1381-1384.

[21] G. Montoro, P. Gilabert, E. Bertran, and J. Berenguer, "A method for real-time generation of slew-rate limited envelopes in envelope tracking transmitters," in RF Front-ends for Software Defined and Cognitive Radio Solutions (IMWS), 2010 IEEE International Microwave Workshop Series on, Feb 2010, pp. 1-4.

[22] P. M. Cheng, O. Garcia, M. Vasic, P. Alou, J. A. Oliver, G. Montoro, and J. A. Cobos, "Envelope amplifier based on a hybrid series converter with the slow-envelope technique," in Energy Conversion Congress and Exposition (ECCE), 2012 IEEE, Sept 2012, pp. 1-5.

[23] A. Khanifar, N. Maslennikov, R. Modina, and M. Gurvich, "Enhancement of power amplifier efficiency through dynamic bias switching," in Microwave Symposium Digest, 2004 IEEE MTT-S International, vol. 3, June 2004, pp. $2047-2050$ Vol.3.

[24] M. Vasic, O. Garcia, J. Oliver, P. Alou, D. Diaz, and J. Cobos, "Multilevel power supply for high efficiency RF amplifiers," in Applied Power Electronics Conference and Exposition, 2009. APEC 2009. Twenty-Fourth Annual IEEE, Feb 2009, pp. 12331238.

[25] Vasić, M.; García, O.; Oliver, J.J.A.; Alou, P.; Diaz, D.; Prieto, R.; Cobos, J.A., "Envelope Amplifier Based on Switching Capacitors for High-Efficiency RF Amplifiers," Power Electronics, IEEE Transactions on, vol.27, no.3, pp.1359,1368, March 2012

[26] Rodriguez, M.; Fernandez-Miaja, P.; Rodriguez, A.; Sebastian, J., "A Multiple-Input Digitally Controlled Buck Converter for Envelope Tracking Applications in Radiofrequency Power Amplifiers," Power Electronics, IEEE Transactions on , vol.25, no.2, pp. 369,381, Feb. 2010

[27] Garcia i Tormo, A.; Poveda, A.; Alarcon, E.; Guinjoan-Gispert, F., "Fundamental Modulation Limits for Minimum Switching Frequency Inband-Error-Free High-Efficiency Power Amplifiers," Circuits and Systems I: Regular Papers, IEEE Transactions on, vol.58, no. 10, pp. 2543,2555 , Oct. 2011

[28] Garcia i Tormo, A.; Poveda, A.; Alarcon, E.; Guinjoan-Gispert, F., "A Study on Multi-Level PWM and Asynchronous /spl Sigma/ /spl Delta/ Modulations for Enhanced Bandlimited Signal Tracking in Switching Power Amplifiers", Circuits and Systems I: Regular Papers, IEEE Transactions on, vol.60, no.6, pp.1621,1634, Apr. 2013

[29] Diaz, D.; Garcia, O.; Oliver, J.A.; Alou, P.; Pavlovic, Z.; Cobos, J.A., "The Ripple Cancellation Technique Applied to a Synchronous Buck Converter to Achieve a Very High Bandwidth and Very High Efficiency Envelope Amplifier," Power Electronics, IEEE Transactions on, vol.29, no.6, pp.2892,2902, June 2014

[30] P F Miaja, A Rodriguez and J Sebastian, "Buck derived converters based on gallium nitride devices for envelope tracking applications," IEEE Trans. Power Electron., vol. 30, no. 4, pp. 2084-2095, Apr. 2015

[31] P. Cheng, M. Vasic, O. Garcia, J. Oliver, P. Alou, and J. Cobos, "Minimum time control for multiphase buck converter: Analysis and application," Power Electronics, IEEE Transactions on, vol. 29, no. 2, pp. 958-967, Feb 2014.

[32] Garcia, O.; Zumel, P.; de Castro, A.; Alou, P.; Cobos, J.A., "Current Self-Balance Mechanism in Multiphase Buck Converter," Power Electronics, IEEE Transactions on, vol.24, no.6, pp.1600,1606, June 2009

[33] Sebastian, J.; Fernandez-Miaja, P.; Rodriguez, A.; Rodriguez, M., "Analysis and Design of the Output Filter for Buck Envelope Amplifiers," Power Electronics, IEEE Transactions on, vol.29, no. 1, pp.213,233, Jan. 2014

[34] D. Diaz, M. Vasic, O. Garcia, J. A. Oliver, P. Alou, and J. A. Cobos, "Hybrid behavioral-analytical loss model for a high frequency and low load dc-dc buck converter," in Energy Conversion Congress and Exposition (ECCE), 2012 IEEE, Sept 2012, pp. $4288-4294$.

[35] Y. Ren, M. Xu, J. Zhou, and F. Lee, "Analytical loss model of power MOSFET," Power Electronics, IEEE Transactions on, vol. 21, no. 2, pp. 310-319, March 2006

[36] Y. F. Wu, A. Saxler, M. Moore, R. Smith, S. Sheppard, P. Chavarkar, T. Wisleder, U. Mishra, and P. Parikh, " $30-w / m m$ GaN HEMTs by field plate optimization," Electron Device Letters, IEEE, vol. 25, no. 3, pp. 117-119, March 2004. 